ipython_galaxy_notebook

August 30, 2016

\title{
1 Maternal Age Effect and Severe Germline bottleneck in the Inheritance of mitochondrial DNA heteroplasmy
}

This notebook replicates the analyses shown in Rebolledo-Jaramillo, Su et al (2014) Maternal Age Effect and Severe Germline bottleneck in the Inheritance of mitochondrial DNA heteroplasmy PNAS October 28, 2014 vol. 111 no. $4315474-15479$

This analysis uses the following datasets as inputs:

- Allele counts produced with Galaxy pipeline (TODO: Provide link to Galaxy history here);

- GenBank file containing sequence and annotation for human mitochondrial genome (accession NC_012920.1);

- Tab-delimited file of ages for individuals analyzed here

- Known list of problematic sites to be excluded from the analysis

\section{Define inputs}

This notebook requires three input datasets: ${ }^{*}$ List of variable sites ${ }^{*}$ List of ages for all individuals ${ }^{*}$ List of bad sites (see "Define problematic sites and regions" below)

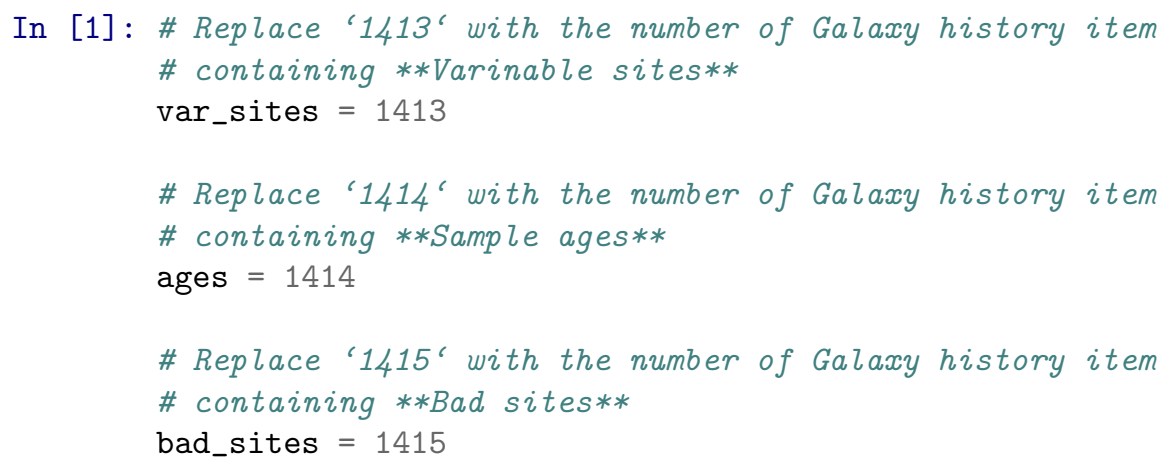

\subsection{Import necessary python modules}

- pandas - A library providing high-performance, easy-to-use data structures and data analysis tools

- numpy - A package for scientific computing with Python

- itertools - Functions for creation of iterators for efficient looping

- biopython - A set of Python modules for biological computation

In [2]: import pandas as pd

import numpy as $\mathrm{np}$

import itertools

from Bio import SeqIO 
from Bio.Seq import Seq

from Bio import Entrez

from Bio.Alphabet import IUPAC

\subsection{Load $R$ extensions and install necessary $R$ modules}

In [3]: \# Load $R$ magic, which will allow running $R$ directly in the notebook \%load_ext rpy2.ipython

In [4]: \# Make a directory where $R$ modules will be installed !mkdir R

mkdir: cannot create directory ' $R$ ': File exists

In [5]: $\%$ R

install.packages ("shape", lib="R", repos="http://cran.cnr.berkeley.edu")

/opt/conda/envs/python2/lib/python2.7/site-packages/rpy2/robjects/functions.py:106: UserWarning: trying

res $=\operatorname{super}\left(\right.$ Function, self).$_{-\_}$call__(*new_args, $* *$ new_kwargs $)$

/opt/conda/envs/python2/lib/python2.7/site-packages/rpy2/robjects/functions.py:106: UserWarning: Conten res $=\operatorname{super}($ Function, self)._call__(*new_args, $* *$ new_kwargs)

/opt/conda/envs/python2/lib/python2.7/site-packages/rpy2/robjects/functions.py:106: UserWarning: lengt

res $=\operatorname{super}($ Function, self)._call__(*new_args, **new_kwargs)

/opt/conda/envs/python2/lib/python2.7/site-packages/rpy2/robjects/functions.py: 106: UserWarning: = res $=\operatorname{super}($ Function, self)._call__(*new_args, **new_kwargs)

/opt/conda/envs/python2/lib/python2.7/site-packages/rpy2/robjects/functions.py:106: UserWarning:

res $=\operatorname{super}($ Function, self)._call__(*new_args, $* *$ new_kwargs $)$

/opt/conda/envs/python2/lib/python2.7/site-packages/rpy2/robjects/functions.py:106: UserWarning: downlo

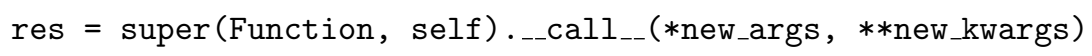

The downloaded source packages are in

'/tmp/RtmpZwnpqq/downloaded_packages'

In [6]: $\% \%$

install.packages ("sm", lib="R", repos="http://cran.cnr.berkeley.edu")

/opt/conda/envs/python2/lib/python2.7/site-packages/rpy2/robjects/functions.py:106: UserWarning: trying

res $=\operatorname{super}($ Function, self)._call__(*new_args, $* *$ new_kwargs $)$

/opt/conda/envs/python2/lib/python2.7/site-packages/rpy2/robjects/functions.py:106: UserWarning: lengt

res $=\operatorname{super}($ Function, self)._call__(*new_args, **new_kwargs)

/opt/conda/envs/python2/lib/python2.7/site-packages/rpy2/robjects/functions.py:106: UserWarning: downlo

res $=\operatorname{super}($ Function, self $) .{ }_{-}$call__- $_{-}(*$ new_args, $* *$ new_kwargs $)$ 
The downloaded source packages are in

'/tmp/RtmpZwnpqq/downloaded_packages'

In $[7]: \% \mathrm{R}$

install.packages ("vioplot", lib="R", repos="http://cran.cnr.berkeley.edu")

/opt/conda/envs/python2/lib/python2.7/site-packages/rpy2/robjects/functions.py:106: UserWarning: trying

res $=\operatorname{super}($ Function, self)._call__(*new_args, **new_kwargs)

/opt/conda/envs/python2/lib/python2.7/site-packages/rpy2/robjects/functions.py:106: UserWarning: lengt

res $=\operatorname{super}($ Function, self)._call__(*new_args, $* *$ new_kwargs)

/opt/conda/envs/python2/lib/python2.7/site-packages/rpy2/robjects/functions.py:106: UserWarning: downlo

res $=\operatorname{super}($ Function, self $) .{ }_{-} \operatorname{call}_{--}(*$ new_args, $* *$ new_kwargs $)$

The downloaded source packages are in

'/tmp/RtmpZwnpqq/downloaded_packages'

In [8]: $\% \mathrm{R}$

require (shape, lib.loc="R")

require (sm, lib.loc="R")

require (vioplot, lib.loc="R")

/opt/conda/envs/python2/lib/python2.7/site-packages/rpy2/robjects/functions.py:106: UserWarning: Loadin

res $=\operatorname{super}($ Function, self)._call__(*new_args, **new_kwargs)

/opt/conda/envs/python2/lib/python2.7/site-packages/rpy2/robjects/functions.py:106: UserWarning: Loadin

res $=\operatorname{super}($ Function, self).__call_-_(*new_args, $* *$ new_kwargs $)$

/opt/conda/envs/python2/lib/python2.7/site-packages/rpy2/robjects/functions.py:106: UserWarning: Packag

res $=\operatorname{super}($ Function, self)._call__(*new_args, $* *$ new_kwargs)

/opt/conda/envs/python2/lib/python2.7/site-packages/rpy2/robjects/functions.py:106: UserWarning: Loadin

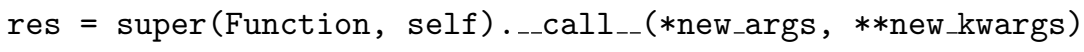

\subsection{Load datasets}

In this example all necessary data are located in Galaxy's history. They can be accessed using the get() function. For example, to load data in the first history item into Jupyter environment simply use get (1), where 1 is the history item number.

Obviously, if your history looks different, change the numbers in the cells below.

In [9]: \# Load Allele Counts

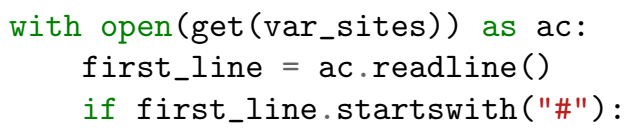




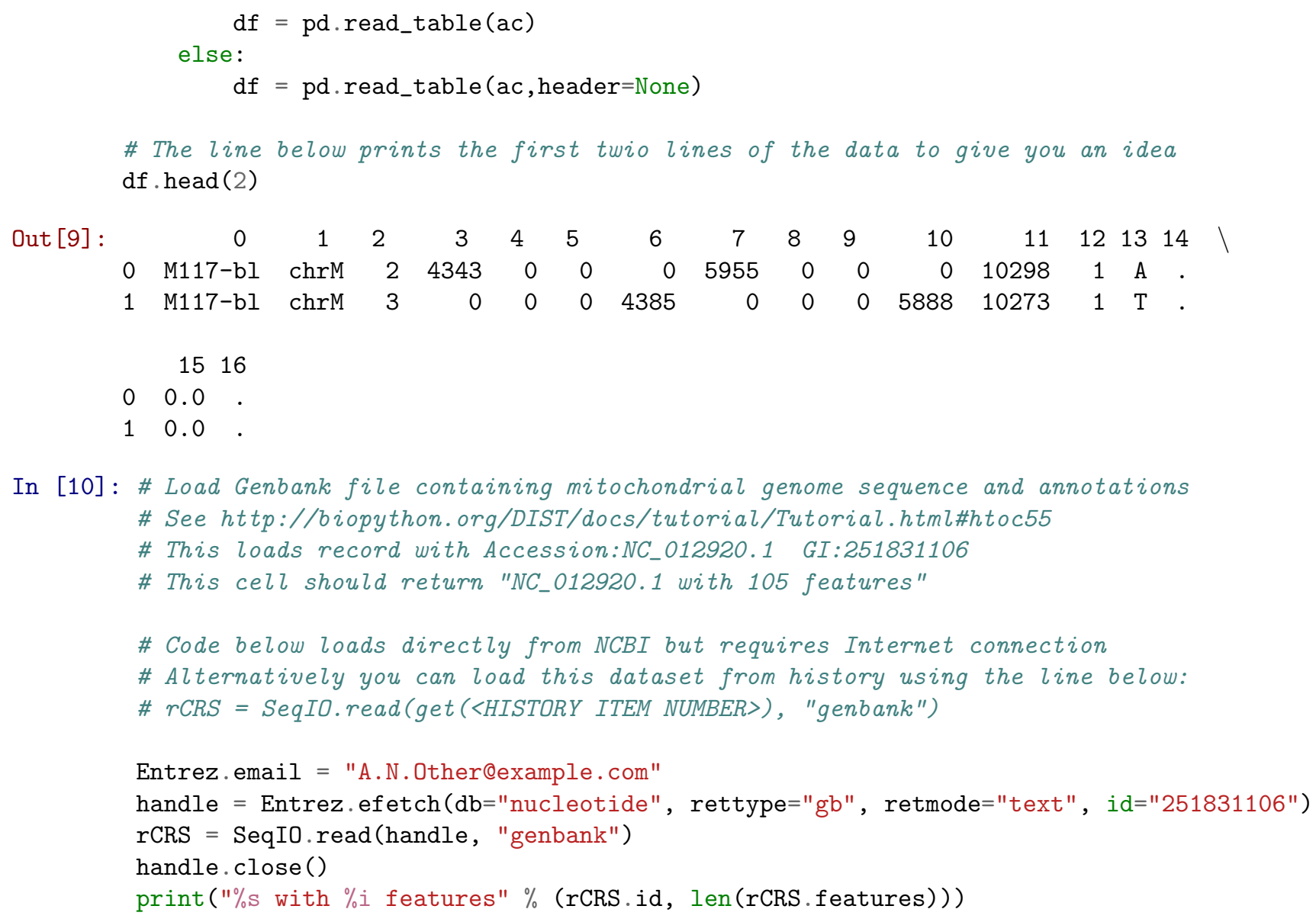

\subsection{Define problematic sites and regions}

- Problematic sites are defined as heteroplasmic sites that failed to be validated by experimental means. In particular, there is an additional screenning step not shown here, where we calculate the cycle bias of the site, i.e. whether the alternative allele is supported primarily by nucleotides within 25 bp of the read ends. There are 9 such cases, and two additional cases of sites we could not replicate with a new long range PCR (deemed PCR errors). These 11 sites are provided as an input dataset for this analysis.

- Problematic reagions include: 
- mtDNA homopolymeres

- region around the artificial "N" at position 3107

- regions within $50 \mathrm{bp}$ of the long range PCR primers

In [12]: \# Read in bad (problematic) sites dataset from history

knownBadhqSites $=$ pd.read_table $($ get $($ bad_sites $)$, header=None $)$

In [13]: \# Define problematic regions

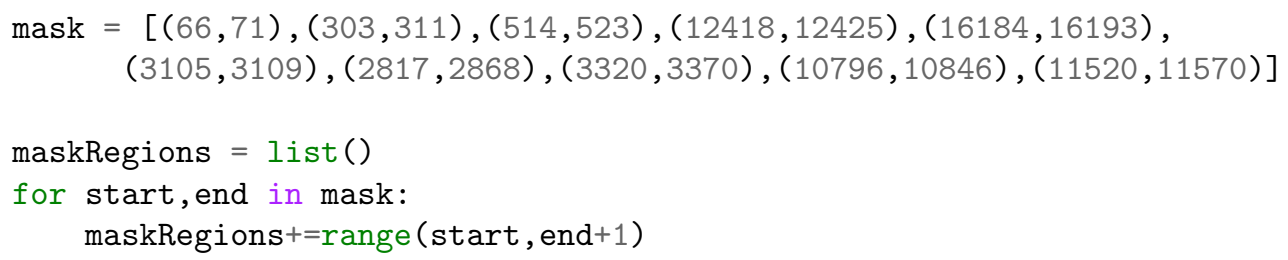

\subsection{Prepare data}

If a header was present in the allele counts input dataset, Pandas assigned the column names automatically. However we will standardize the column names so they can be easily accessed later.

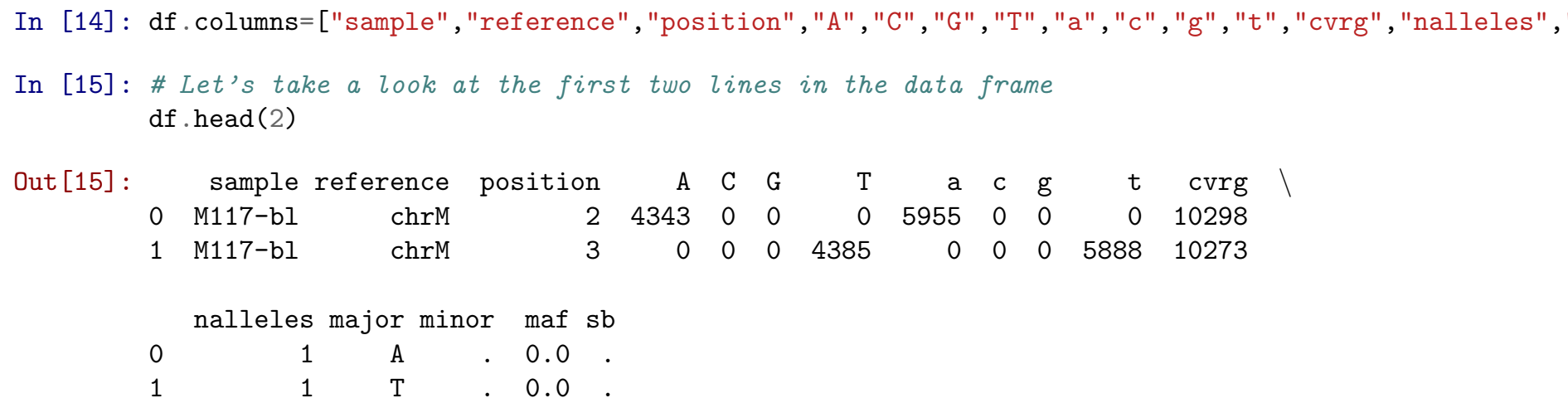

In our data, all but one mother-child pair conforms to the naming convention:

\begin{tabular}{ll}
\hline mother & child \\
\hline family-tissue & familyChild\#-tissue \\
M477-ch & M477C1-ch \\
\hline
\end{tabular}

However, the pair M502G (grandmother) and M501 (mother) break the rule. So, we adjusted their ids accordingly:

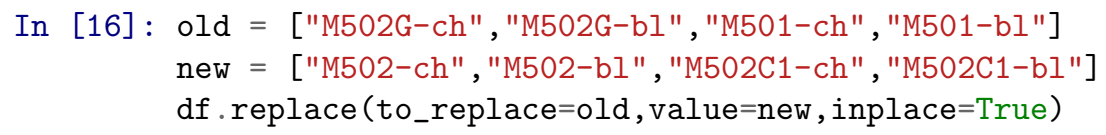

\subsection{Plot sequencing depth distribution (Fig. S7)}

At this point we can calculate the coverage distribution of each sample, as shown in Figure S7 in the PNAS paper. To do so, we need to split the dataframe into blood and cheek dataframes, and make the object available to $\mathrm{R}$ (via Rpy2). 
In [17]: \# Here we split the dataframe into blood and cheek samples

blood $=\operatorname{df}[\mathrm{df}[$ 'sample'].str.contains $("-\mathrm{bl} ")]$

cheek $=\operatorname{df}[\mathrm{df}[$ 'sample'].str. contains $("-\operatorname{ch} ")]$

In [18]: \# Let's look at blood data frame

blood.head (2)

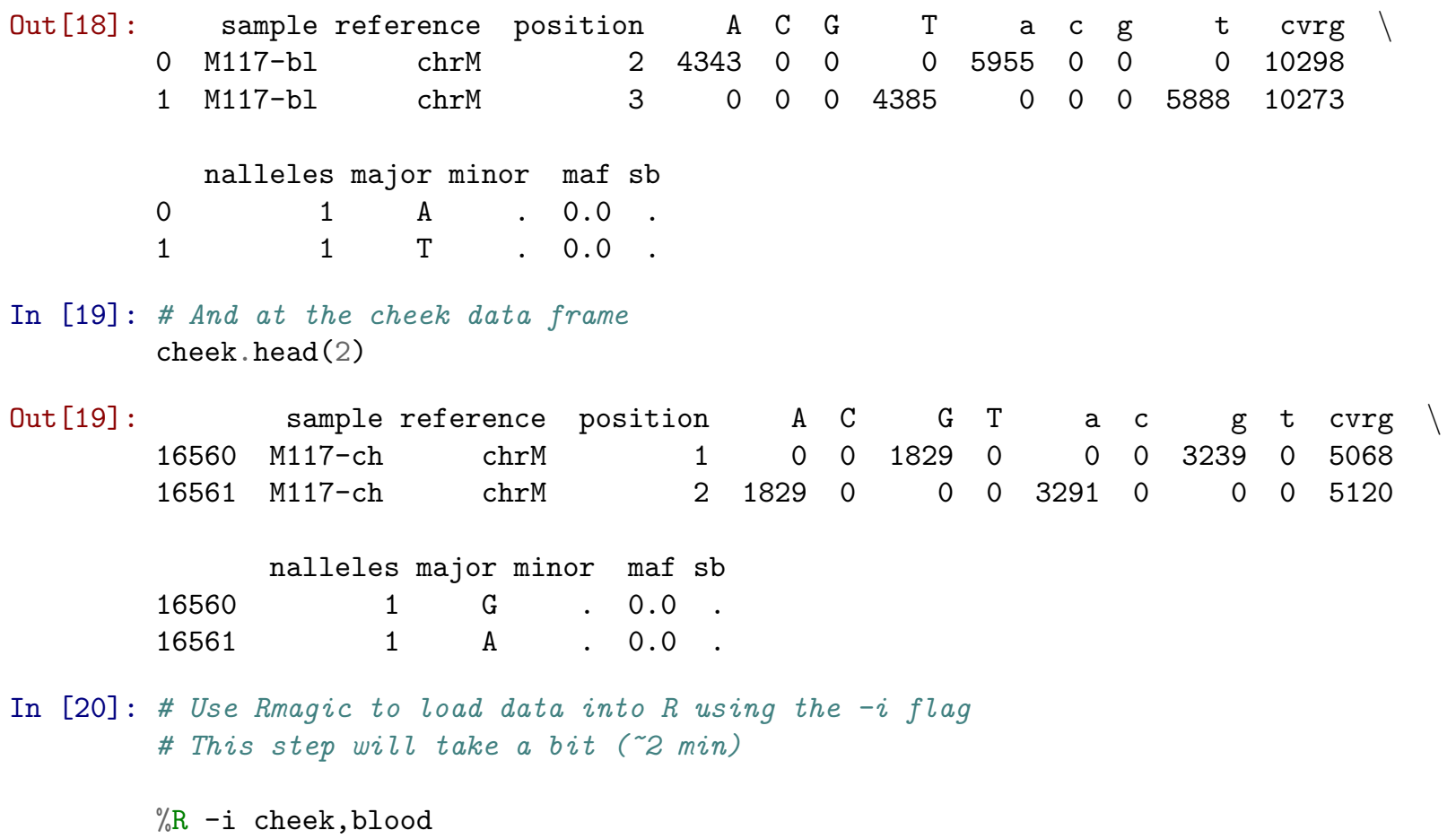

Let's peek at the $\mathrm{R}$ version of the blood dataframe:

In $[21]: \% \mathrm{R}$

head (blood, 2)

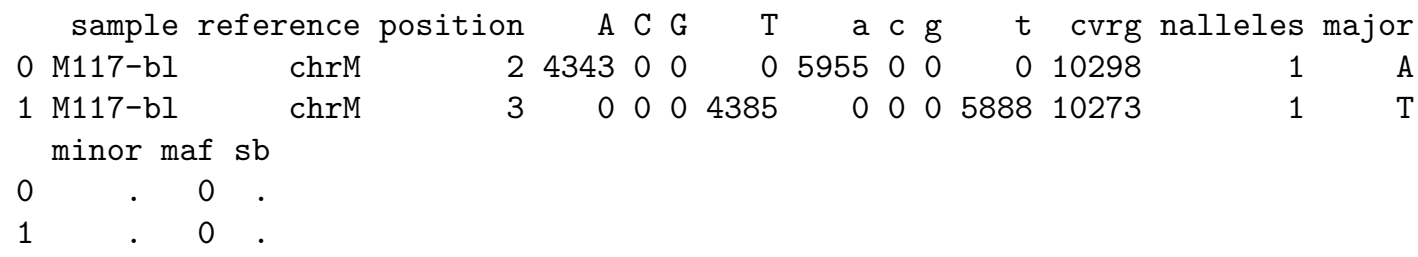

Transform numeric looking columns into actual numeric columns to guarantee the value types:

In $[22]: \% \%$

tonumeric $=c(3: 13,16)$

blood [,tonumeric] = apply(blood[,tonumeric], 2, function(x) as.numeric $(\operatorname{as.character}(\mathrm{x})))$ $\operatorname{cheek}[, \operatorname{tonumeric}]=\operatorname{apply}(\operatorname{cheek}[, \operatorname{tonumeric}], 2, \operatorname{function}(\mathrm{x})$ as.numeric $(\operatorname{as} \cdot \operatorname{character}(\mathrm{x})))$

Define custom R function to generate Figure S7:

In $[23]: \% \mathrm{R}$

boxPlotCvrg = function(data,tissue) \{ 


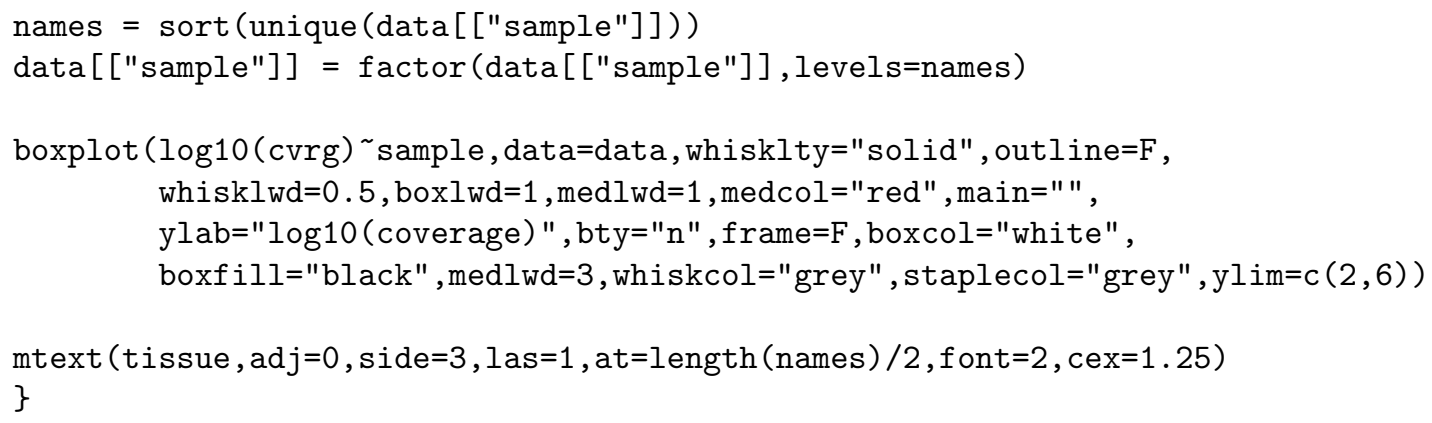

\subsubsection{Plot the figure}

You can adjust the size of the plotting image by adjusting:

- $-\mathrm{w}=$ width

- $-\mathrm{h}=$ height

- $-\mathrm{u}=$ units

- $-\mathrm{r}=$ resolution

In [24]: $\% \%$-w 18 -h $10-\mathrm{u}$ in $-\mathrm{r} 72$
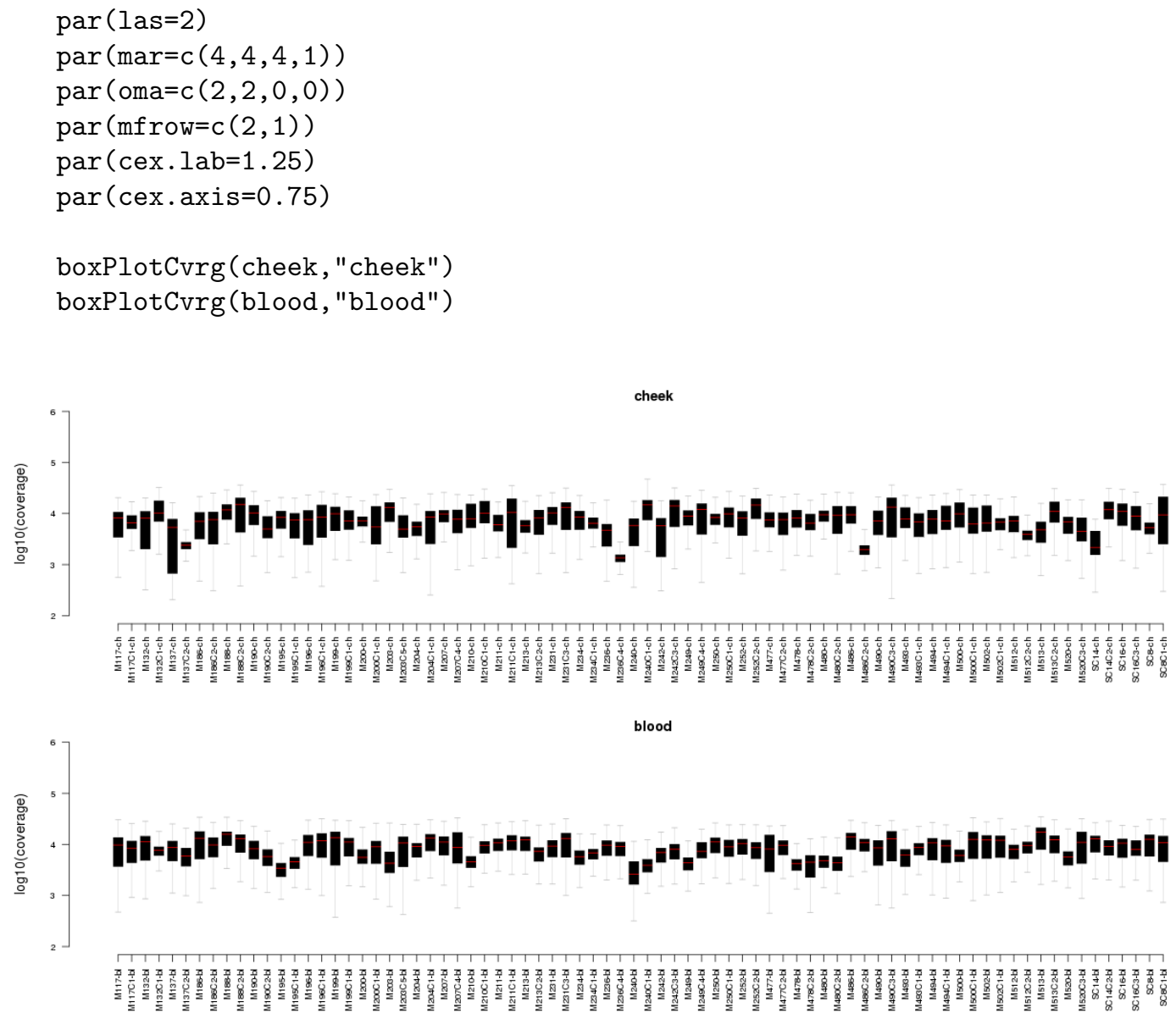


\subsection{Define high quality heteroplasmic sites}

We define high quality (HQ) sites as:

1. minor allele frequency (maf) $\geq 1 \%$

2. coverage $\geq 1000$

3. maf balance ( $1 \%$ in forward and reverse strands)

4. no strand bias

5. outside "problematic sites":

- mtDNA homopolymeres

- around the artificial "N" at position 3107

- within $50 \mathrm{bp}$ of the long range PCR primers

In [25]: \# Filter sites on minor allele frequncy (maf), coverage (cvrg) and wheather \# the sites are located in problematic regions

\# The list of problematic region (maskRegions) is defined in cell 12 above hq_sites $=\operatorname{df}[(\mathrm{df} \cdot \operatorname{maf}>=0.01)$ \& (df.cvrg $>=1000)$ \& $\sim \mathrm{df} \cdot$ position.isin(maskRegions) $]$

In [26]: len(hq_sites)

Out [26] : 559

By applying these initial filters, we reduced the dataframe from 2 million lines to $\mathbf{5 7 2}$ lines only, which is much more manageable. Next, we calculate strand bias and maf balance for these 572 sites. The strand bias calciulation is perfomed according to Guo Y et al. 2012

In [27]: \# Compute strand and minor allele frequency bias

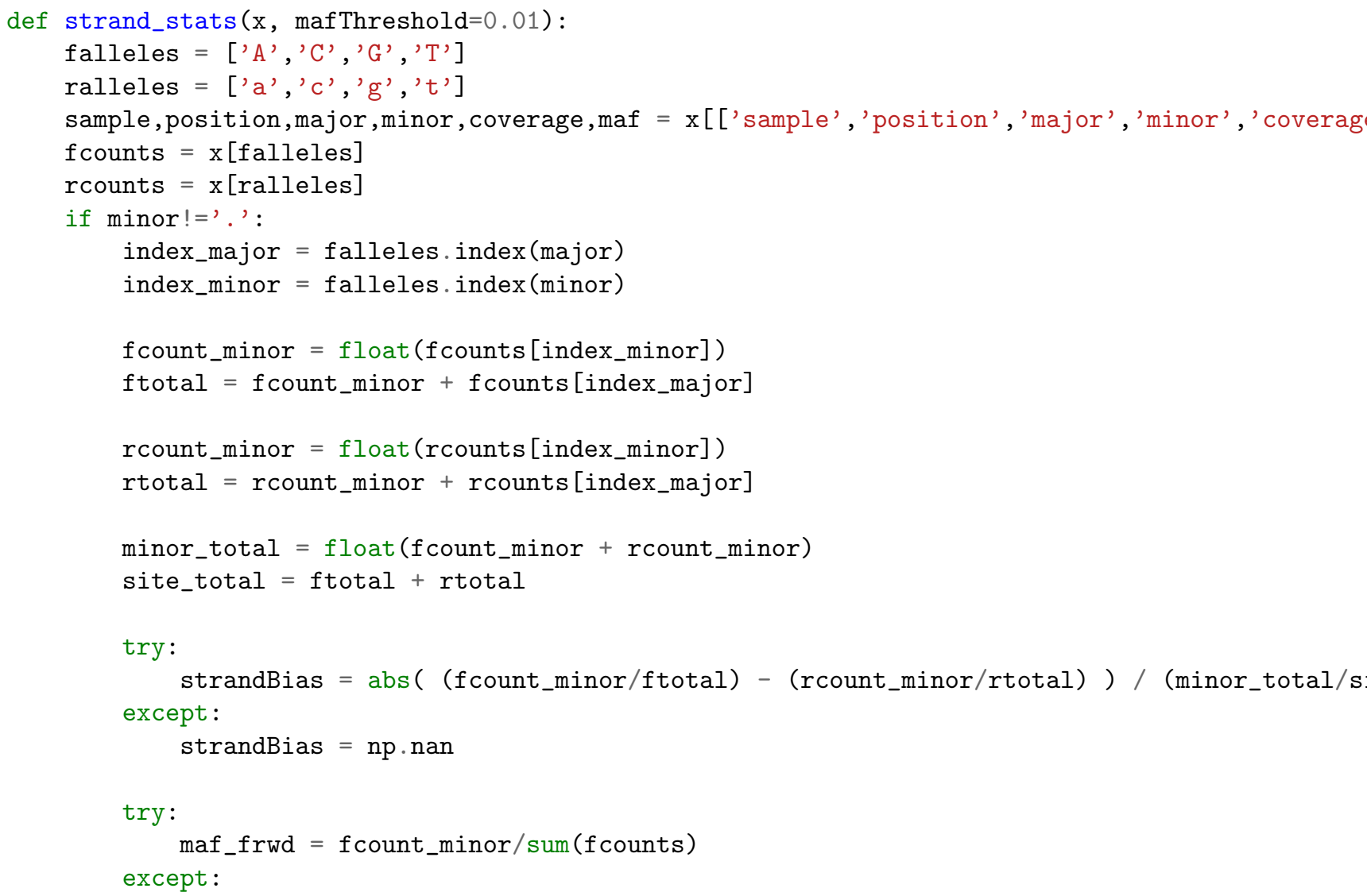




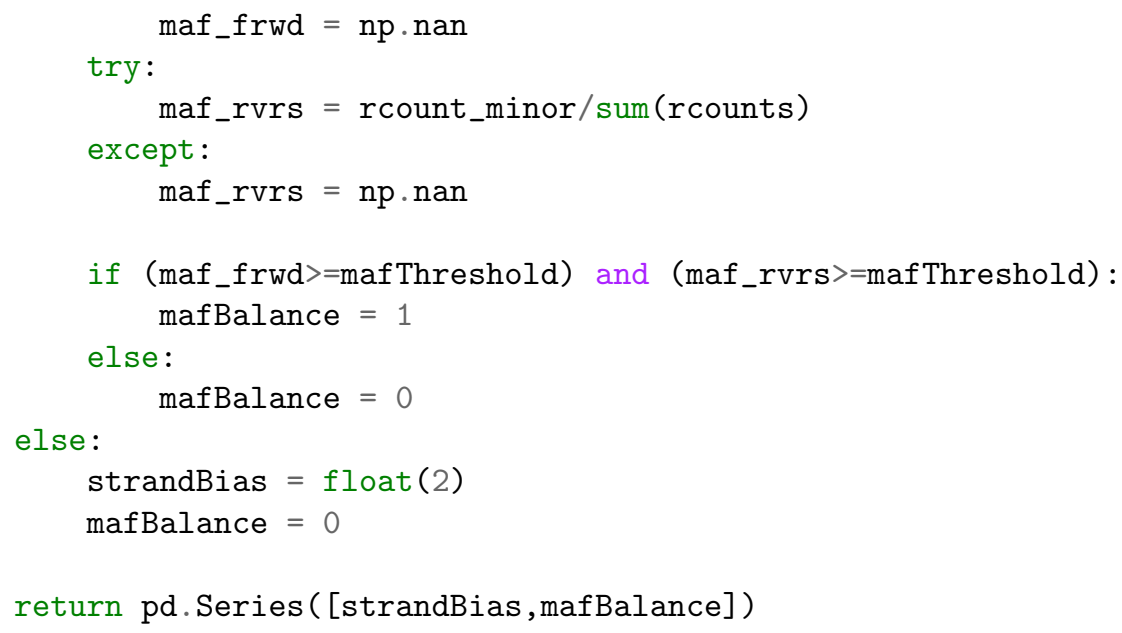

\subsection{Test statistical significance of high quality sites}

Finally, we calculate the significance of the minor allele frequency of a site provided the error rate at that position. The error rate is estimated from the remaining 155 samples, and the expected allele acounts are compared to the observed allele counts:

In [32]: from scipy.stats import poisson 
In [33]: \# We define a poisson function that will take a single high quality site, and explore the vari \# the position among the remaining samples

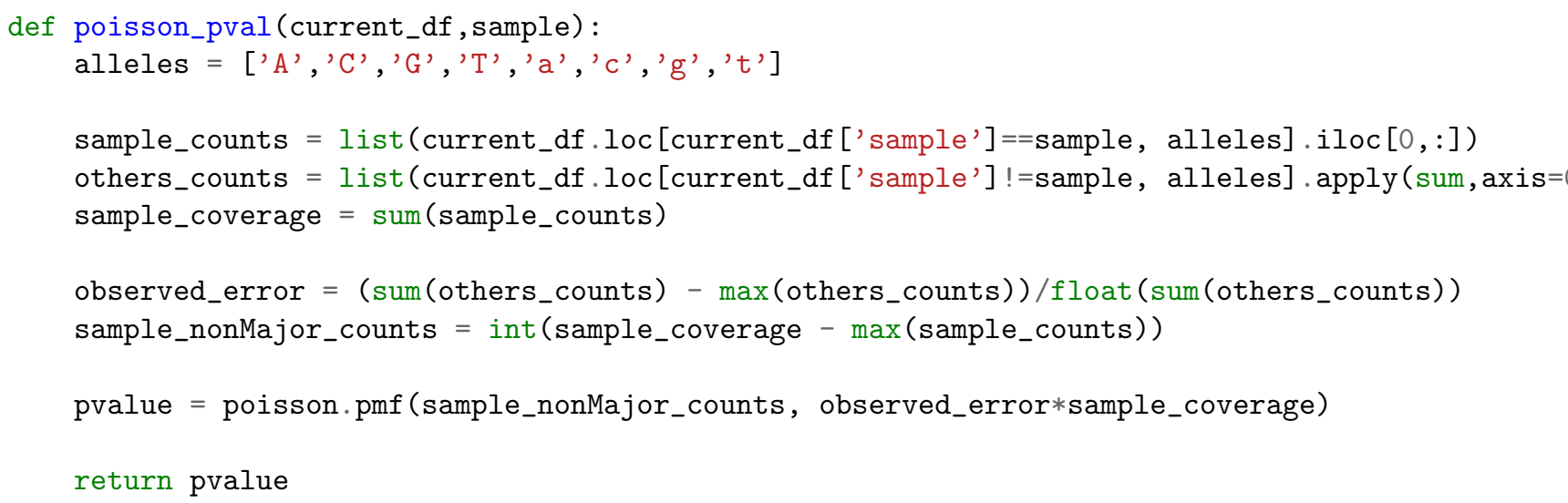

Out [34]: 181

As described in the paper, all sites were statiscally significant under the Poisson and Likelihood (not shown here) frameworks.

\section{Screening for contamination}

In our previous publication, Dickins, Rebolledo-Jaramillo, et al (2014) Controlling for contamination in resequencing studies with a reproducible web-based phylogentic approach BioTechniques, 56(3):134-141, we described warning signs of a potential contamination. They include: 1 . Excess heteroplasmic sites ( $\geq 5$ per sample) 2. Tight minor allele frequency distribution 3. Non-family related positions of heteroplasmic sites

We routinely apply our contamination detection pipeline, so we are confident our sites in the PNAS paper were not artifacts. As an example of the screening for contamination, we can plot the number of sites and the minor allele frequency distribution of all samples in the high quality sites set:

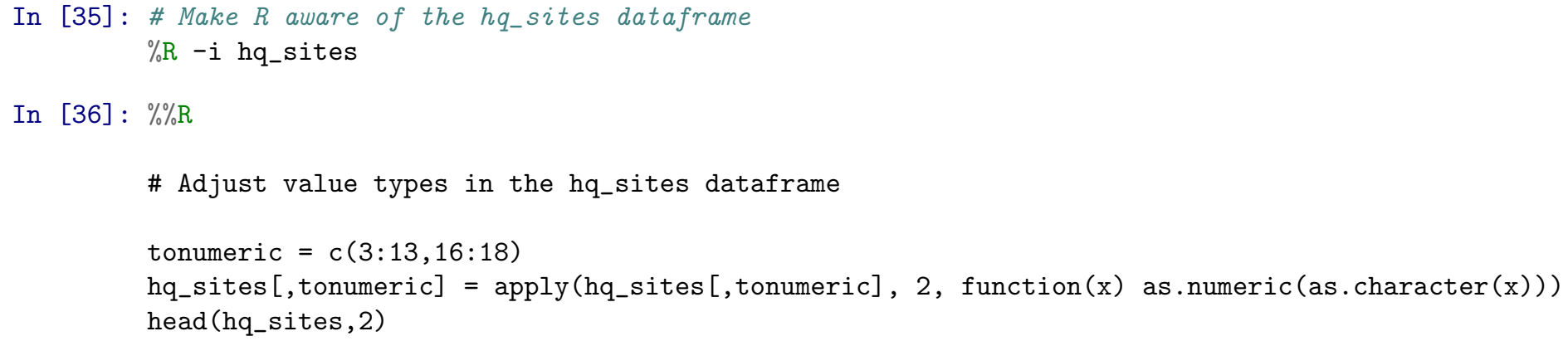




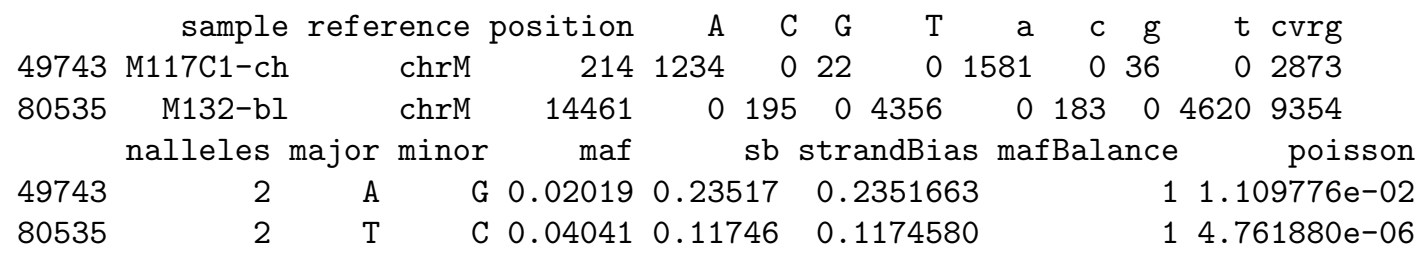

In [37]: $\% \%$-w $10-\mathrm{h} 15-\mathrm{u}$ in $-\mathrm{r} 72$

\# Plot hq_sites number of sites and minor allele frequency distribution

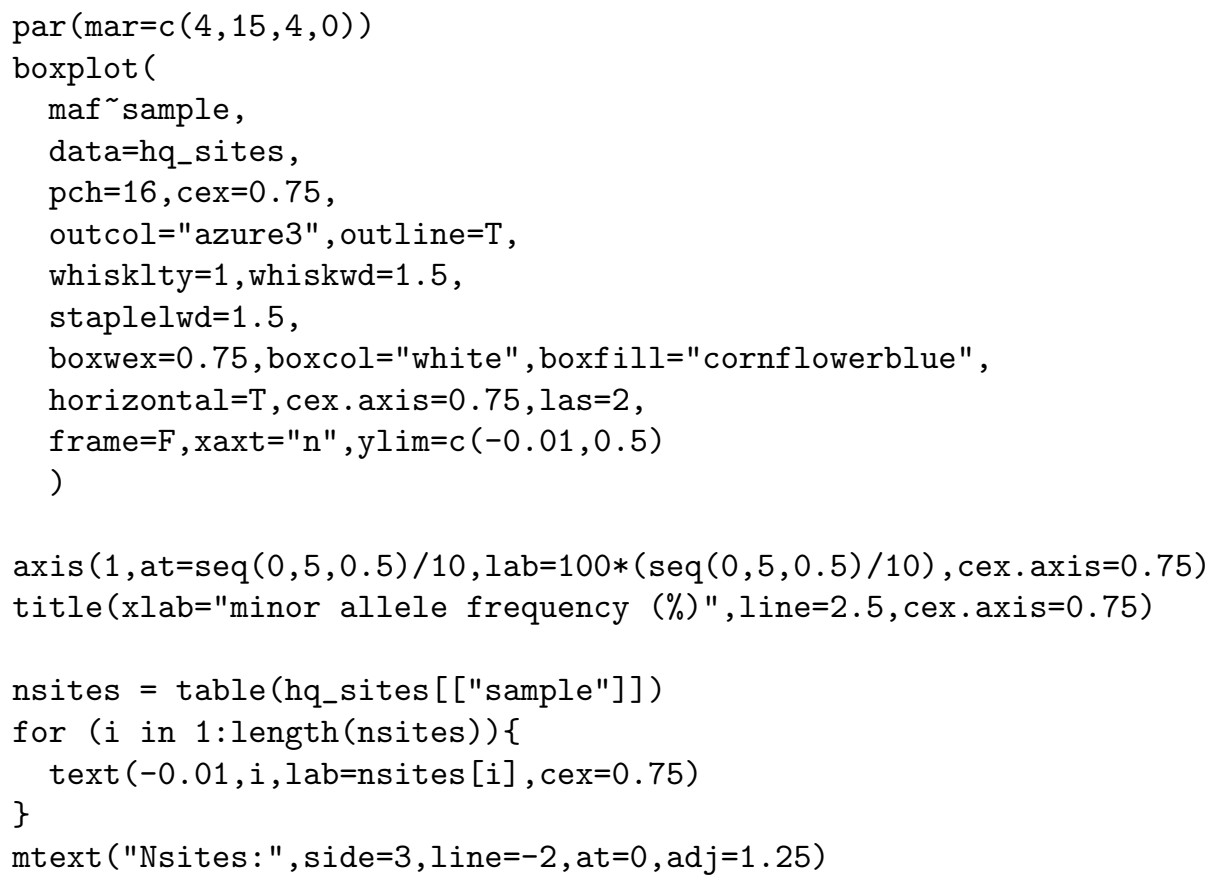




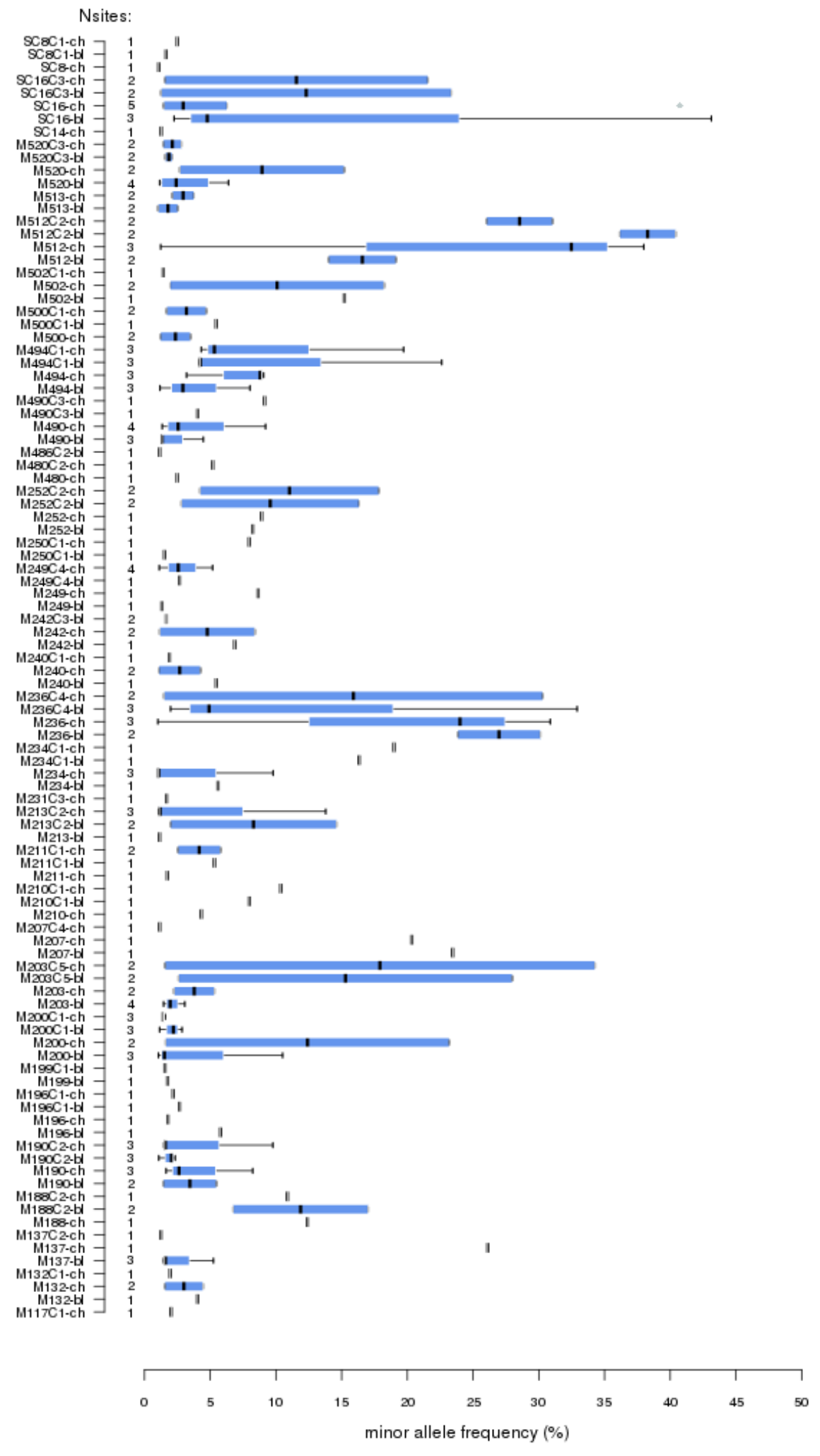




\subsection{Placing high quality sites into quartets}

For each high quality site, we can retrieve the minor allele frequency information for the remaining 3 samples in the family collection. So, a quartet is simply a tabulation of the minor allele frequency for the mother blood and cheek, and her child blood and cheek, for the same site. Below is an example of a quarted for family M494:

\begin{tabular}{llllllll}
\hline family & position & major & minor & mother_cheek & mother_blood & child_cheek & child_blood \\
\hline M494 & 9196 & G & A & 0.032 & 0.030 & 0.000 & 0.000 \\
\hline
\end{tabular}

However, before we can do that, we need to add family information to the high quality sites. We will do that by extracting the family id from each sample's id. It is also useful to have a way to split the data by tissue or member of the pair, so we will add the columns family, tissue and member, accordingly.

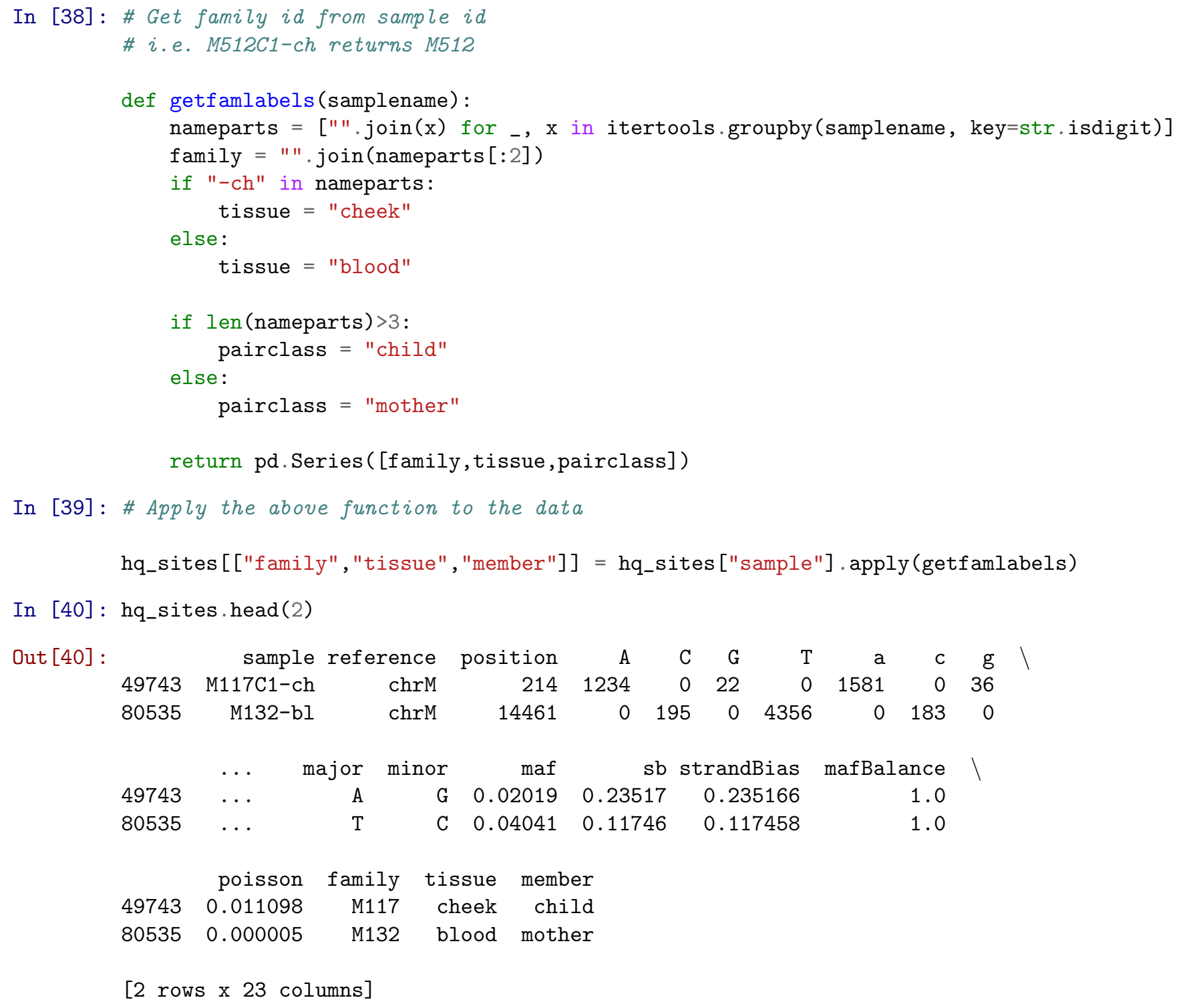


In [41]: \# Now we can extract the unique quartets by selecting the "family" and "position" columns

unique_quartets = hq_sites[["family", "position"]].drop_duplicates()

len(unique_quartets)

Out $[41]: 108$

In [42]: unique_quartets.head(2)

Out [42]: family position

$80535 \quad$ M132 14461

In [43]: \# For each family_id/position combination,

\# retrieve the information for all 4 members of the quartets

\# from the original data dataframe

def getQuartets(hqsite):

position = hqsite['position']

familyid = hqsite['family']

pos_data $=\operatorname{df}[\mathrm{df}[$ 'position'] == position $]$

allmembers $=$ [s for $s$ in pos_data['sample'].drop_duplicates() if s.startswith(familyid) ] mother $=\min ([\operatorname{len}(\mathrm{x})$ for $\mathrm{x}$ in allmembers $])$

child $=\max ([\operatorname{len}(\mathrm{x})$ for $\mathrm{x}$ in allmembers $])$

if len(allmembers) $==4$ :

for member in allmembers:

if len (member)==mother and member .endswith ("-ch"):

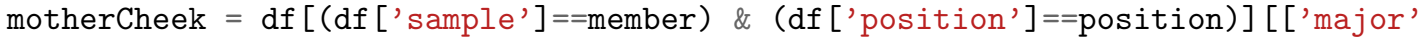

elif len(member)==mother and member.endswith ("-bl"):

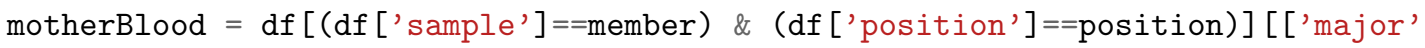

elif len (member)==child and member endswith ("-ch"):

childCheek $=\operatorname{df}[(\mathrm{df}[$ 'sample']==member) \& (df ['position']==position)][['major', else:

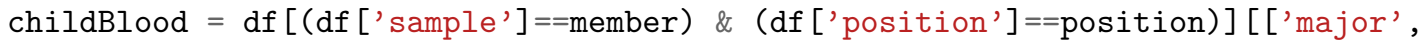

return pd.Series([familyid,position]+list(motherCheek)+list (motherBlood)+list(childChe else:

pass

In [44]: \# Apply getQuartets to data

quartets = unique_quartets.apply (getQuartets, axis=1)

In [45]: \# The following is necssary to remove empty rows from the dataframe quartets = quartets.dropna()

In [46]: quartets

Out [46] :

49743

$\begin{array}{llll}0 & 1 & 2 & 3\end{array}$

456

$\begin{array}{lll}7 & 8 & 9\end{array}$

$\begin{array}{lll}10 & 11 & 12\end{array}$

80535

214.0 A G

0.00181 A G

$\begin{array}{llllll}0.00043 & A & G & 0.02019 & A & G\end{array}$

$\begin{array}{llllllllllllll}M 132 & 14461.0 & \mathrm{~T} & \mathrm{C} & 0.04461 & \mathrm{~T} & \mathrm{C} & 0.04041 & \mathrm{~T} & \mathrm{C} & 0.00145 & \mathrm{~T} & \mathrm{C}\end{array}$

82828

M132 185.0 A $\quad$ G 0.01535 A $\quad$ G

0.00569 A $\quad$ G 0.00522 A G 


\begin{tabular}{|c|c|c|c|c|c|c|c|c|c|c|c|c|c|}
\hline & 32 & 4.0 & C & 1 & 307 & C & 1 & 30 & & . & & & \\
\hline 0360 & 137 & 953.0 & A & & 000 & A & c & 1451 & & & & & \\
\hline 325 & 137 & 3918.0 & $\mathrm{~T}$ & c & 05 & & & & & & & & \\
\hline 727 & 137 & 320.0 & C & & 120 & & & 245 & & & & & \\
\hline 1703 & 137 & 054.0 & C & & 18 & c & & & & & & & \\
\hline 8891 & 188 & 16240.0 & A & & 103 & A & c & & & & & & \\
\hline 8908 & 190 & 3202.0 & $\mathrm{~T}$ & & 1641 & $\mathrm{~T}$ & c & 420 & & & & & \\
\hline 0811 & 190 & 5105.0 & $\mathrm{~T}$ & & .02625 & $\mathrm{~T}$ & ( & .05490 & & & 000 & & \\
\hline 2450 & 190 & 215.0 & A & & .08234 & A & G & 0.00097 & & c & 22 & A & \\
\hline 5134 & 190 & 6379.0 & $\mathrm{~T}$ & & .00000 & $\mathrm{~T}$ & c & 056 & & & 85 & & \\
\hline 6061 & 190 & 7306.0 & $\mathrm{~T}$ & C & .00032 & $\mathrm{~T}$ & & 0.00049 & & & 73 & & \\
\hline 5236 & 190 & 16482.0 & A & c & 153 & A & G & 033 & & & & & \\
\hline 4020 & 196 & 6172.0 & $\mathrm{~T}$ & & 780 & $\mathrm{~T}$ & C & 783 & & & & & \\
\hline 7120 & 196 & 6274.0 & G & A & 021 & G & $A$ & 038 & & & & & \\
\hline 22 & 199 & 16150.0 & C & $\mathrm{T}$ & 227 & C & 7 & 761 & & & & & \\
\hline 55 & 99 & 1747.0 & G & $\mathrm{T}$ & & G & A & 035 & & & & & \\
\hline 62 & 200 & 596.0 & $\mathrm{~T}$ & & & $\mathrm{~T}$ & & & & & & & \\
\hline 46 & 00 & 8584.0 & G & & & G & A & & & & & & \\
\hline 33 & 00 & 1.0 & $\mathrm{C}$ & $\mathrm{T}$ & & C & $\mathrm{T}$ & 60 & & & & & \\
\hline & 0 & 98.0 & G & A & & G & A & 062 & & & & G & \\
\hline & 0 & 4.0 & A & & & A & G & & & & & A & \\
\hline & & 1.0 & C & $\mathrm{T}$ & & C & $\mathrm{T}$ & & & & & & \\
\hline & & 2.0 & $\mathrm{~T}$ & C & & $\mathrm{T}$ & C & & & & & 1 & \\
\hline & & & G & & & G & & & & & & & \\
\hline & & & $\mathrm{T}$ & & & $\mathrm{T}$ & & & & & & 1 & \\
\hline & & & A & & & & G & & & & & $\mathrm{A}$ & \\
\hline 568 & 203 & .0 & $\mathrm{~T}$ & & & $\mathrm{~T}$ & C & 07 & $\mathrm{~T}$ & & & $\mathrm{~T}$ & \\
\hline & & & & & & & & & & & & & \\
\hline & & & C & $\mathrm{T}$ & & $C$ & $\mathrm{~T}$ & & C & & & C & \\
\hline & & & $\mathrm{T}$ & & & $\mathrm{T}$ & & & & & & $\mathrm{T}$ & \\
\hline & & & A & & & A & G & & A & & & A & \\
\hline & & & $\mathrm{T}$ & & & $\mathrm{T}$ & & & & & & $\mathrm{T}$ & \\
\hline & & & A & & & A & & & & & & A & \\
\hline & & & & & & & & & & & & A & \\
\hline & & & & & & & & & & & & A & \\
\hline & & & A & & & A & c & & & & & 1 & \\
\hline & 502 & & A & & & A & c & & & & & 1 & \\
\hline & & & A & $c$ & & A & c & & & & & & \\
\hline & & & G & & & G & 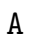 & & & & & A & \\
\hline & & & & & & A & c & & & & & & \\
\hline & & & & & & $T$ & 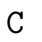 & & & & & & \\
\hline & & & c & & & G & 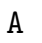 & & & & & & \\
\hline & & & & & & A & 0 & & & & & & \\
\hline & & 0 & G & & & G & & & & & & & \\
\hline & & & $\mathrm{T}$ & & & $T$ & & & & & & & \\
\hline & & & & & & $T$ & & & & & & & \\
\hline & & & $\mathrm{T}$ & & & C & & & & & & & \\
\hline & & & A & & & A & & & & & & & \\
\hline & M52 & & A & & & & & & & & & & \\
\hline & & & $\mathrm{T}$ & & & $\mathrm{T}$ & & & & & & & \\
\hline & & & c & & & & & & & & & & \\
\hline & & 3.0 & $\mathrm{~T}$ & & & $\mathrm{~T}$ & C & & 7 & & & $\mathrm{~T}$ & \\
\hline & & 2.0 & C & & & & . & & $c$ & & & & \\
\hline & & 49.0 & S & A & 0.02945 & & $A$ & 0.02249 & G & & 0.00015 & G & \\
\hline
\end{tabular}




$\begin{array}{rrrrrrrrrrrrrr}2502781 & \text { SC16 } & 16170.0 & \text { A } & \text { G } & 0.06300 & \text { A } & \text { G } & 0.04775 & \text { A } & \text { G } & 0.00031 & \text { A } & \text {. } \\ 2503365 & \text { SC16 } & 185.0 & \text { A } & \text { G } & 0.01450 & \text { A } & \text { G } & 0.00908 & \text { A } & \text { G } & 0.00182 & \text { A } & \text { G } \\ 2512743 & \text { SC16 } & 9565.0 & \text { G } & \text { A } & 0.01445 & \text { G } & \text { A } & 0.01051 & \text { G } & \text { A } & 0.00095 & \text { G } & \text { A } \\ 2519899 & \text { SC16 } & 152.0 & \text { T } & \text { C } & 0.00178 & \text { T } & \text { C } & 0.00059 & \text { T } & \text { C } & 0.01532 & \text { T } & \text { C }\end{array}$

$\begin{array}{lr} & 13 \\ 49743 & 0.00215 \\ 80535 & 0.00089 \\ 82828 & 0.00506 \\ 115798 & 0.00019 \\ 140360 & 0.00000 \\ 145325 & 0.00000 \\ 147727 & 0.00036 \\ 191703 & 0.00599 \\ 308891 & 0.06739 \\ 328908 & 0.00013 \\ 330811 & 0.00000 \\ 342450 & 0.00000 \\ 365134 & 0.02330 \\ 366061 & 0.01087 \\ 375236 & 0.02052 \\ 474020 & 0.00044 \\ 507120 & 0.02656 \\ 539922 & 0.00050 \\ 558655 & 0.01555 \\ 589762 & 0.00158 \\ 597746 & 0.00000 \\ 602733 & 0.00049 \\ 623866 & 0.01150 \\ 631050 & 0.02196 \\ 634146 & 0.02859 \\ 649833 & 0.00044 \\ 666023 & 0.00037 \\ 666180 & 0.00183 \\ 666832 & 0.00062 \\ 666887 & 0.00043 \\ \ldots & \ldots \\ 1975402 & 0.22615 \\ 2000080 & 0.04190 \\ 2012839 & 0.04255 \\ 2046767 & 0.00127 \\ 2046777 & 0.00027 \\ 2067319 & 0.05447 \\ 2090706 & 0.00028 \\ 2113750 & 0.00053 \\ 2156362 & 0.00141 \\ 2163961 & 0.40417 \\ 2166255 & 0.36141 \\ 2179847 & 0.00040 \\ 2228303 & 0.00055 \\ 2242245 & 0.00451 \\ 2245112 & 0.00044 \\ 2291624 & 0.00117 \\ 2293155 & 0.00047\end{array}$




$\begin{array}{ll}2298590 & 0.00126 \\ 2307452 & 0.00672 \\ 2326004 & 0.01572 \\ 2329629 & 0.02127 \\ 2382193 & 0.00017 \\ 2402442 & 0.01640 \\ 2444730 & 0.00049 \\ 2488965 & 0.23353 \\ 2497760 & 0.00025 \\ 2502781 & 0.00000 \\ 2503365 & 0.00053 \\ 2512743 & 0.00046 \\ 2519899 & 0.01251\end{array}$

[105 rows $\mathrm{x} 14$ columns]

In [47]: \# Set column names

\# mc: mother cheek

\# mb: mother blood

\# cc: child cheek

\# cb: child blood

quartets. columns = ["family", "position", "mcMajor", "mcMinor", "mcMAF", "mbMajor", "mbMinor", "mbMAF "ccMajor", "ccMinor", "ccMAF", "cbMajor", "cbMinor" , "cbMAF"]

In [48]: quartets.head(2)

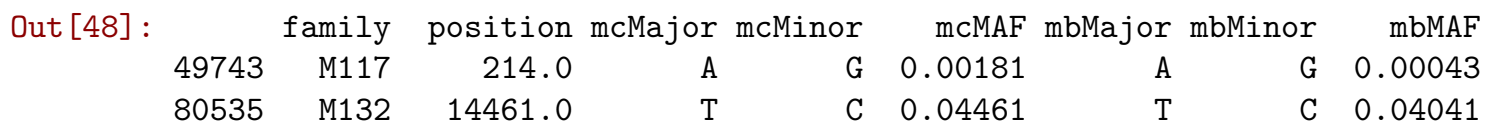

$\begin{array}{rrrrrrr} & \text { ccMajor } & \text { ccMinor } & \text { ccMAF } & \text { cbMajor } & \text { cbMinor } & \text { cbMAF } \\ 49743 & \mathrm{~A} & \mathrm{G} & 0.02019 & \mathrm{~A} & \mathrm{G} & 0.00215 \\ 80535 & \mathrm{~T} & \mathrm{C} & 0.00145 & \mathrm{~T} & \mathrm{C} & 0.00089\end{array}$

We can add even more information to the quartets table. For instance, the impact of the alternative allele and the nucleotide change class:

In [49]: \# Define function for generating protein translation

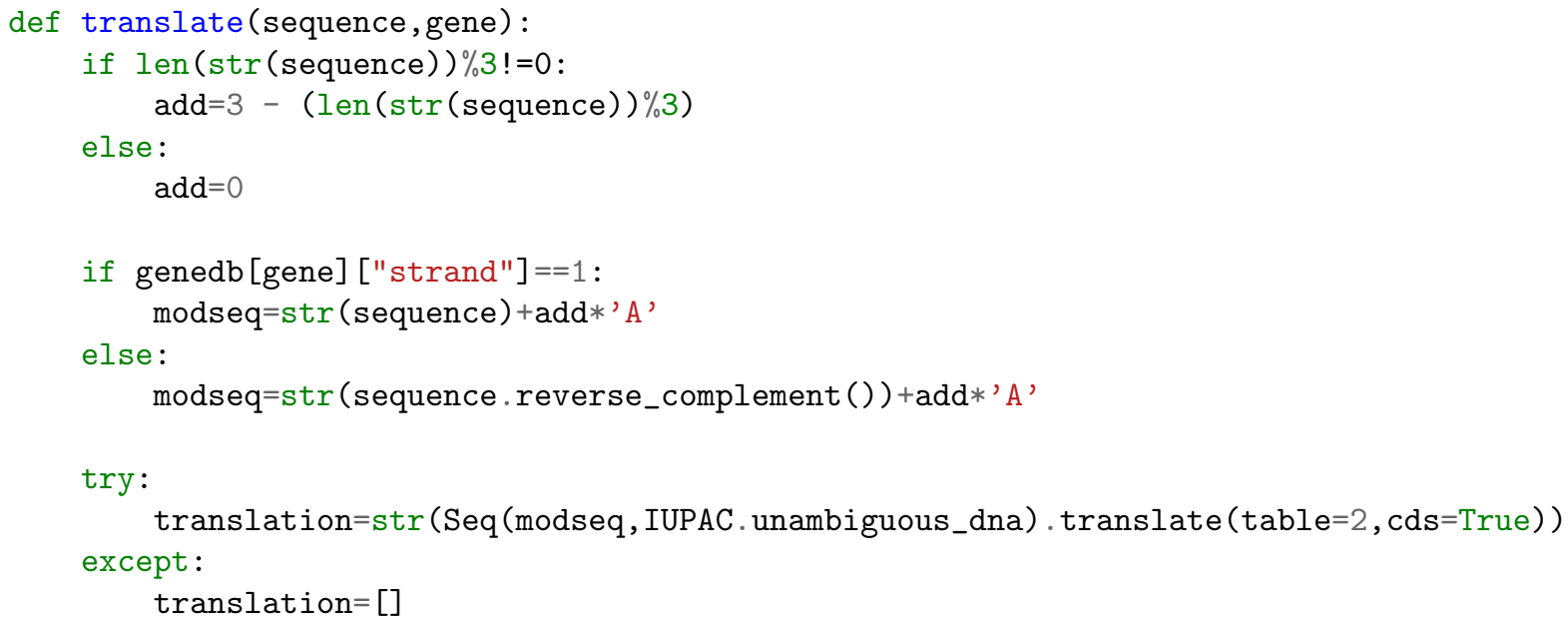


return translation

In [50]: \# Define function for estimating evolutionary impact

\# The function determines if a heteroplamic site

\# synonymous/non-synonomous and if the change is transitional or transversional

def evoImpact(quartet):

try:

het, major, minor $=$ quartet

pos $=$ int (het) -1

gene $=[\mathrm{g}$ for $\mathrm{g}$ in genedb if genedb[g] ['end'] $>=$ pos $>=$ genedb [g] ['start'] [0]

if gene in [feature.qualifiers['gene'][0] for feature in rCRS.features if feature.type

majorseq $=$ rCRS.seq. tomutable ()

minorseq $=$ rCRS.seq.tomutable $($ )

$\operatorname{majorseq}[\mathrm{pos}]=\operatorname{major}$

minorseq[pos] = minor

ref_seq = rCRS.seq[genedb[gene] ["start"]:genedb[gene] ["end"]]

major_seq $=$ majorseq[genedb[gene] ["start"]:genedb[gene] ["end"]]

minor_seq $=\operatorname{minorseq}[$ genedb $[$ gene] ["start"] :genedb[gene] ["end"]

if (translate (ref_seq,gene)==translate (minor_seq,gene)) :

ptimpact $=$ "syn"

else:

ptimpact $=$ "nonsyn"

else:

ptimpact $="-"$

$\mathrm{ntClass}=\{$ 'pu': ['A', 'G'], 'py': ['C', 'T']\}

majorClass $=[\mathrm{k}$ for $\mathrm{k}, \mathrm{v}$ in ntClass.iteritems () if major in $\mathrm{v}]$

minorClass $=[\mathrm{k}$ for $\mathrm{k}, \mathrm{v}$ in ntClass.iteritems () if minor in $\mathrm{v}]$

if majorClass $==$ minorClass:

ntimpact=' ts'

else:

ntimpact =' $t v '$

return pd.Series ([ptimpact, genedb[gene] ['class'],ntimpact])

except :

pass

In [51]: \# Using BioPython rCRS object defined in cell 10

\# Parse mitochondrial genome features

genedb $=\operatorname{dict}()$

labs = ["class", "start", "end", "strand"]

for feature in rCRS.features:

if feature.type in ["rRNA", "tRNA", "CDS"] :

ftype $=$ feature.type 


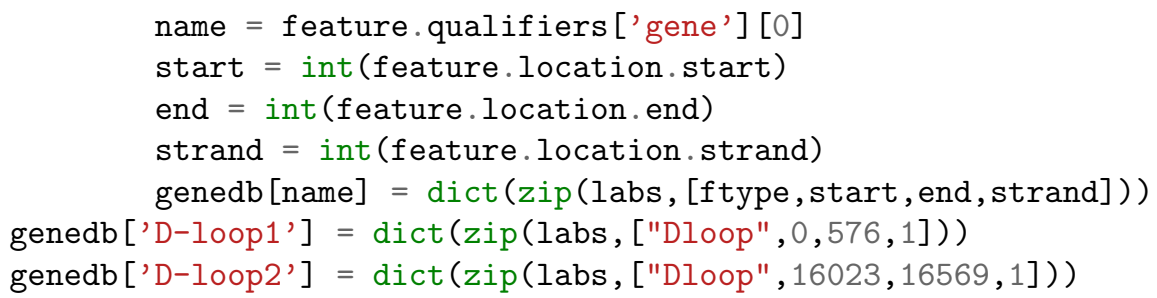

In [52]: a = quartets.1oc [282115:328908]

a.head (20)

\#a[['position', 'mbMajor', 'mbMinor']].apply (evoImpact, axis=1)

Out [52]:

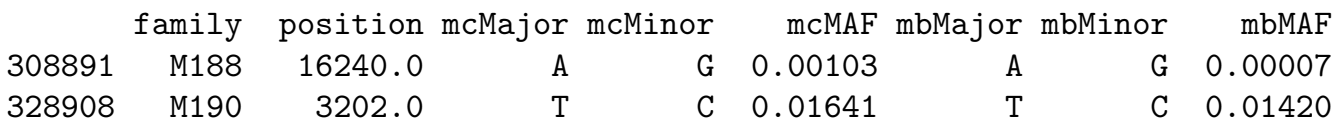

$\begin{array}{rrrrrrr} & \text { ccMajor } & \text { ccMinor } & \text { ccMAF } & \text { cbMajor } & \text { cbMinor } & \text { cbMAF } \\ 308891 & \text { A } & \text { G } & 0.10872 & \text { A } & \text { G } & 0.06739 \\ 328908 & \text { T } & \text { C } & 0.00043 & \text { T } & \text { C } & 0.00013\end{array}$

In [53]: \# We set the ancestral state to the alleles found in the mother's blood sample.

quartets [["ptchange", "class", "ntchange"]] = quartets [['position', 'mbMajor', 'mbMinor']. apply(e

Finalized quartets table:

In [54]: quartets.head(2)

\begin{tabular}{|c|c|c|c|c|c|c|c|c|c|}
\hline Out [54]: & & family & position & mcMajor & mcMinor & mcMAF & mbMajor & mbMinor & mbMAF \\
\hline & 49743 & M117 & 214.0 & A & G & 0.00181 & A & G & 0.00043 \\
\hline & 80535 & M132 & 14461.0 & $\mathrm{~T}$ & $\mathrm{C}$ & 0.04461 & $\mathrm{~T}$ & C & 0.04041 \\
\hline & & ccMajor & ccMinor & CCMAF & cbMajor & cbMinor & CbMAF & ptchange & class \\
\hline & 49743 & $\mathrm{~A}$ & G & 0.02019 & A & G & 0.00215 & - & Dloop \\
\hline & 80535 & $\mathrm{~T}$ & C & 0.00145 & $\mathrm{~T}$ & $\mathrm{C}$ & 0.00089 & nonsyn & CDS \\
\hline & & ntchange & & & & & & & \\
\hline & 49743 & ts & & & & & & & \\
\hline & 80535 & ts & & & & & & & \\
\hline
\end{tabular}

\subsection{Plot the number of heteroplasmic sites per individual or family (Fig. S11)}

In [55]: \# Since we modified the hq_sites dataframe, we have to reload it in $R$

$\% R$-i hq_sites, quartets

In $[56]: \% R$

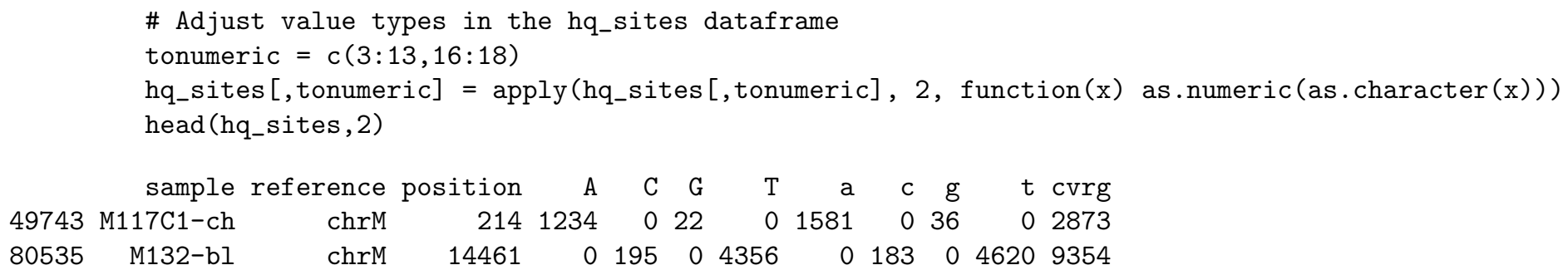




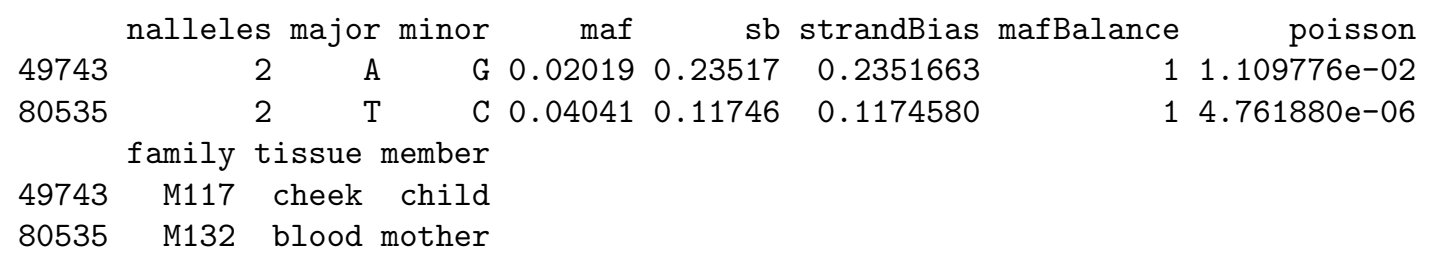

In $[57]: \% \mathrm{R}$

\# Frequency (number of sites per individual)

getFreq $=$ function(data, tissue, member $)\{$

siteFreq $=$ data.frame $($ table $($ table (as.character $($ data $[$ (data $[$ ["tissue"] $]==t i s s u e) \&$ (data $[[" m$

siteFreq $=$ unlist (apply (siteFreq, $1, F U N=f u n c t i o n(x) \operatorname{rep}(x[1], x[2])))$

siteFreq $=$ as.numeric $(c(r e p(0,39-$ length $($ siteFreq $))$, siteFreq $))$

return (siteFreq)

\}

In $[58]: \% \%$

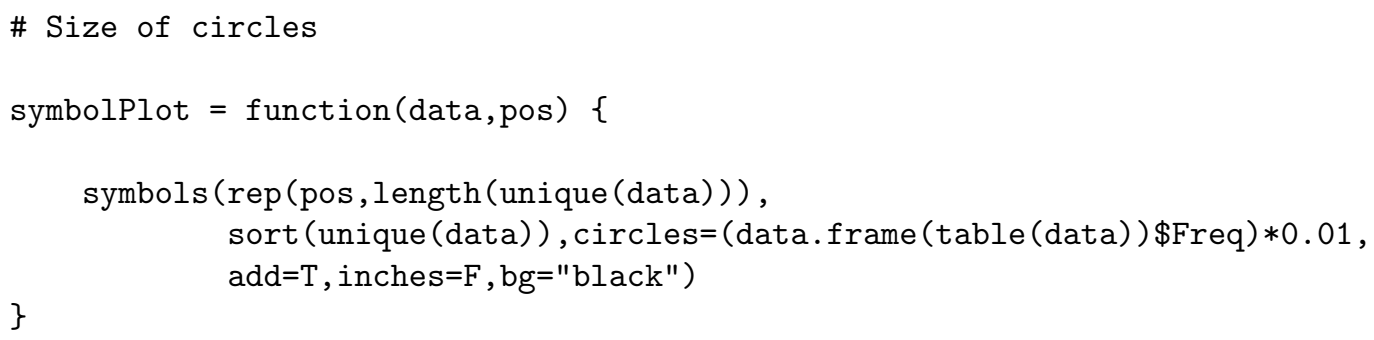

In $[59]: \% \%$

\# Backbone boxplot

boxPlotNsites = function(data,pos, addOpt="False") \{

boxplot (data, $y \mathbf{l i m}=c(-2$, maxSites $)$, frame $=F$, axes $=F, x l i m=c(1,7)$, at $=p o s, \operatorname{col}=r g b(0,0,0,0)$, boxlwd=2, boxcol=" coral3", medcol="coral3", whisklty="solid", whiskcol=" coral3", staplecol="coral3", add=as.logical (addOpt), outline=F)

\}

In [60]: $\% \%$ - w $11-\mathrm{h} 8-\mathrm{u}$ in $-\mathrm{r} 72$

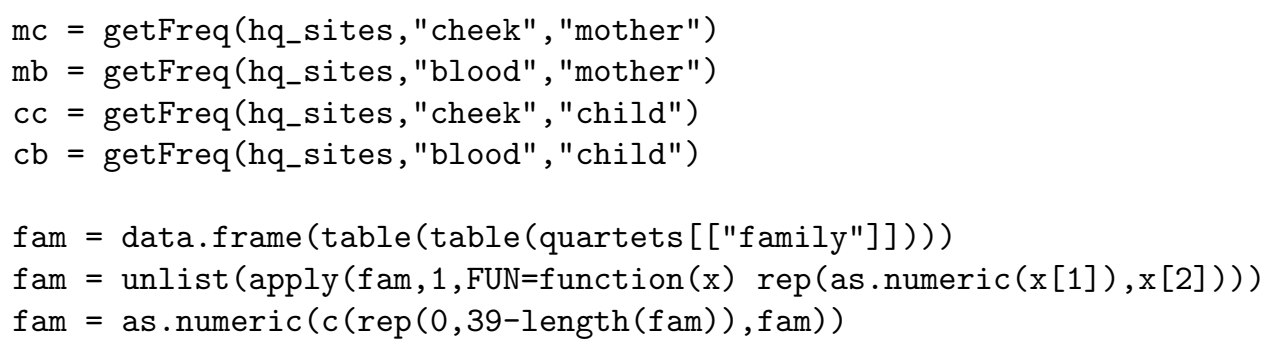



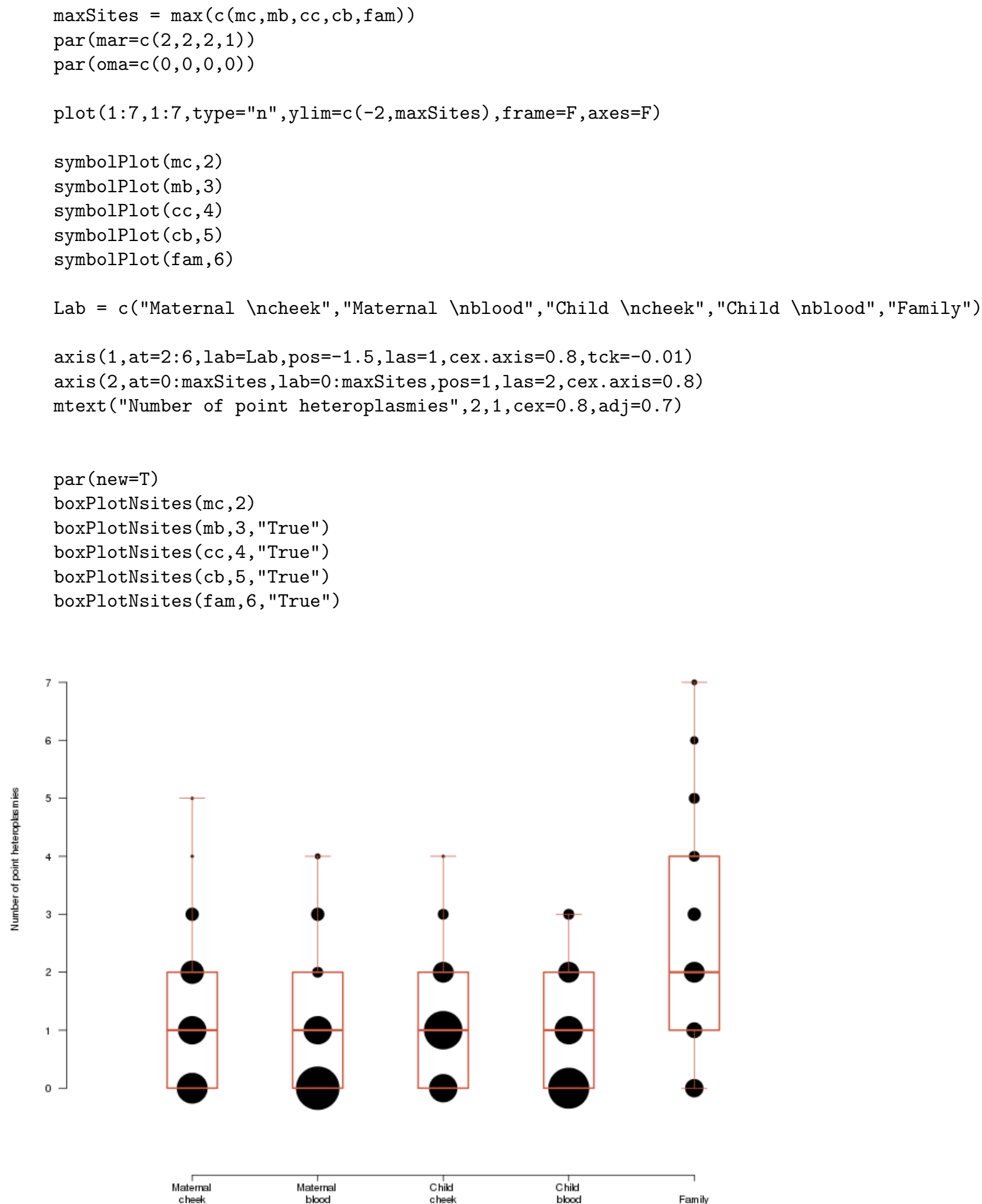

Child
cheek

Child
bbod

blood

Family 


\subsection{Plot distribution of high quality heteroplasmies (Fig. S10)}

In $[61]: \%$ R

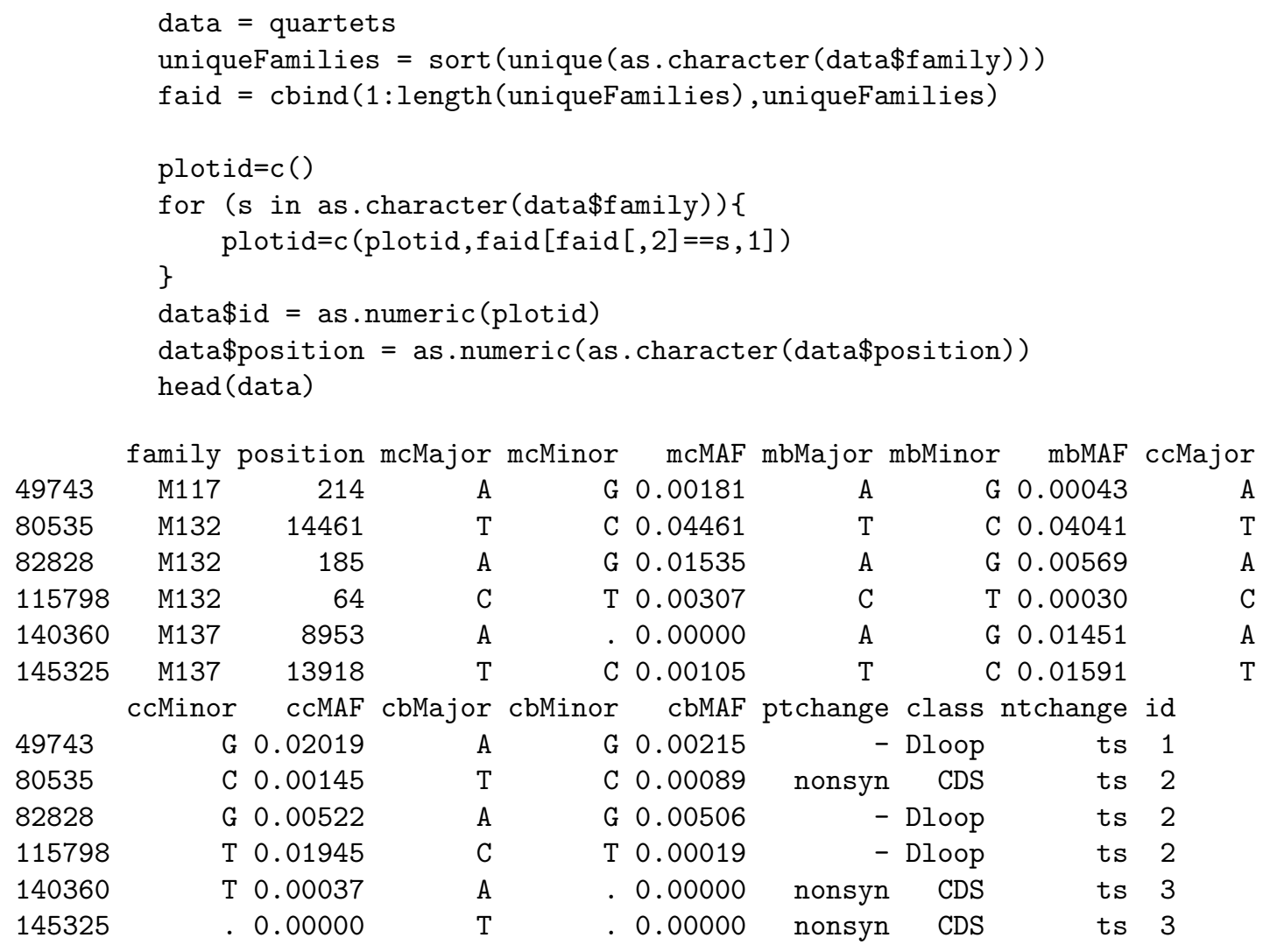

In $[62]: \% \%$

\# Define colors for each mitochondrial genome features

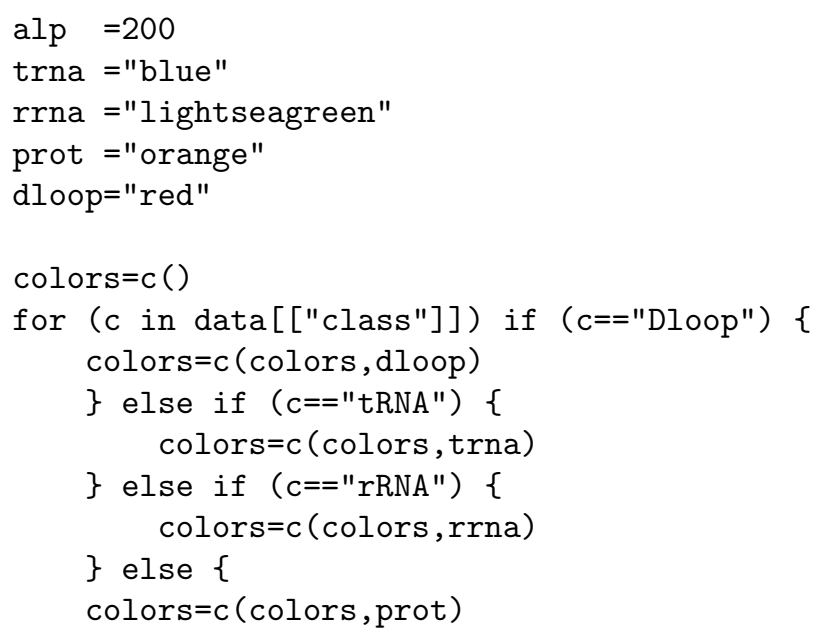


In $[63]: \% \%$

\# Define symbols depending on whether a site is syn/nonsyn or ts/tv

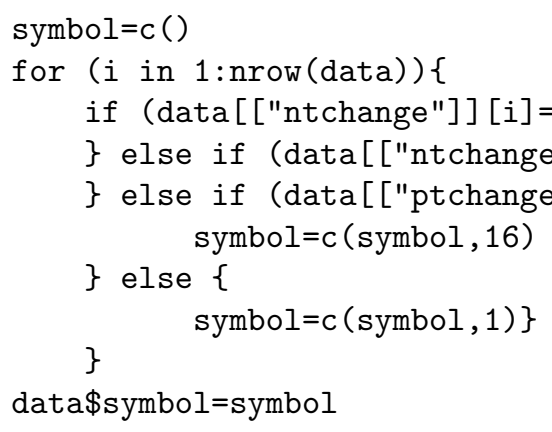




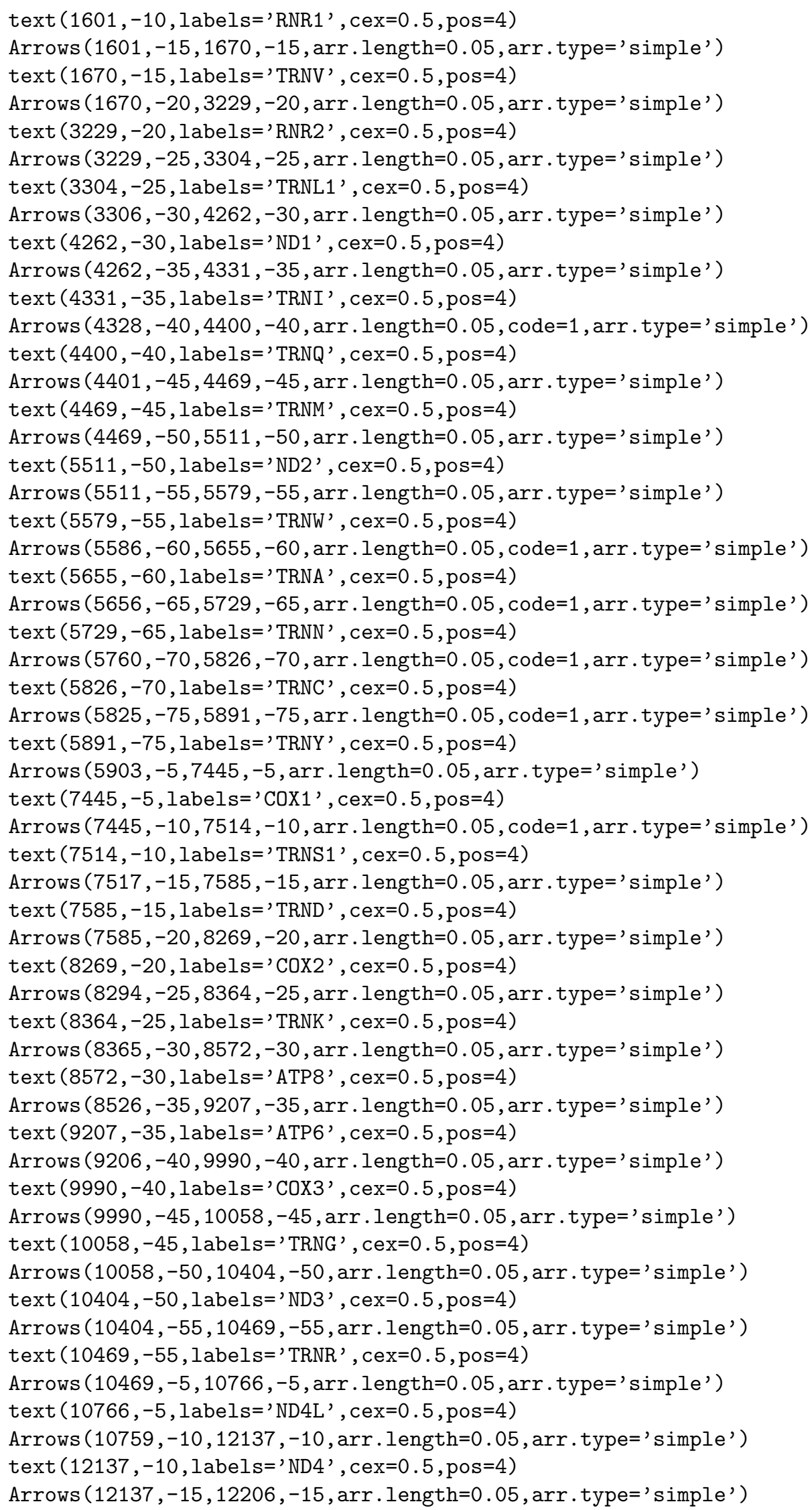



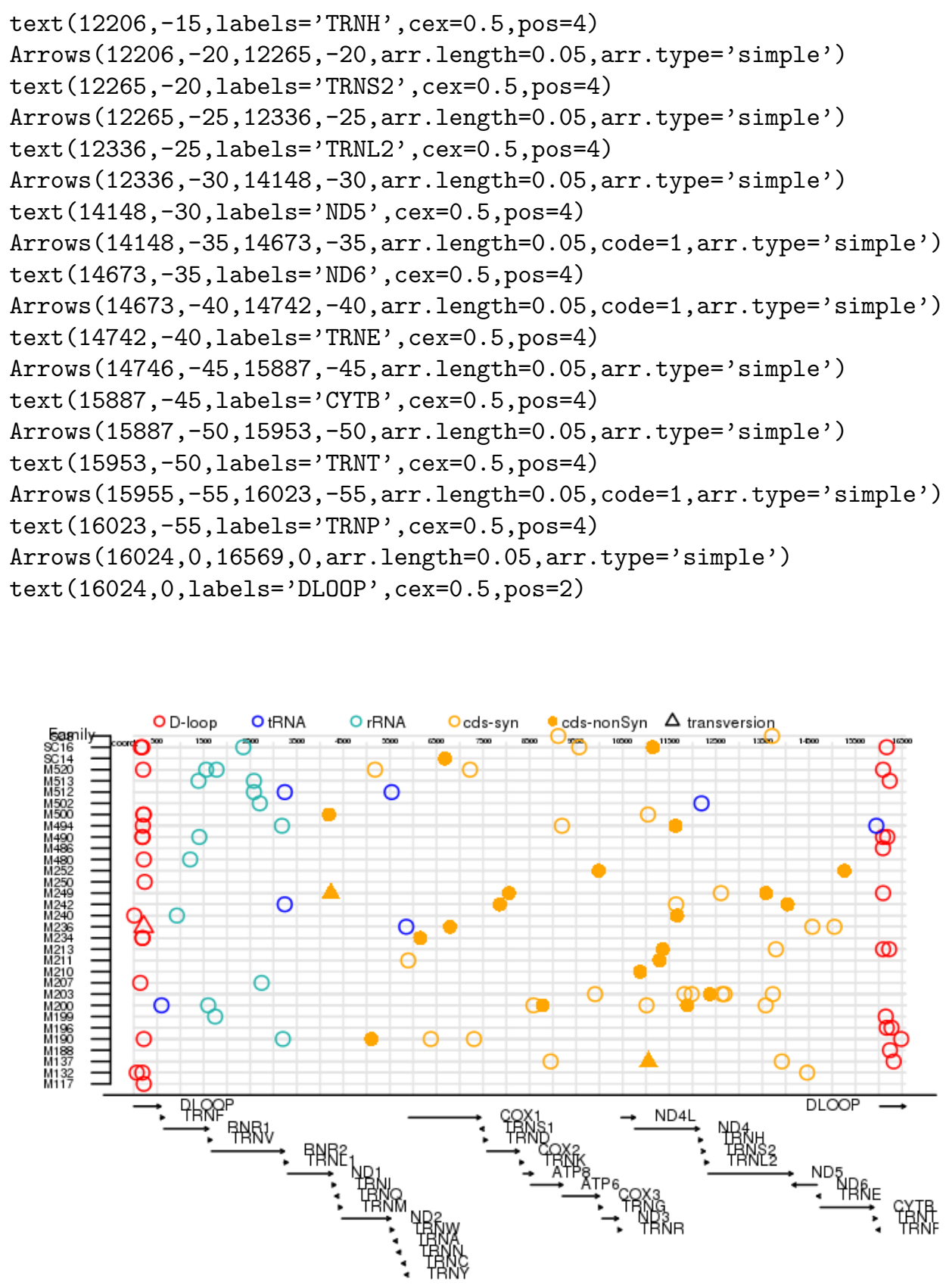

\subsection{Plot correlations in minor allele frequencies (Fig. 1)}

In $[65]: \% \mathrm{R}$

\#head (quartets, 3)

quartets [as. character (quartets\$cbMinor)! =as . character (quartets\$ccMinor), ]

family position mcMajor mcMinor mcMAF mbMajor mbMinor mbMAF ccMajor 


\begin{tabular}{|c|c|c|c|c|c|c|c|c|}
\hline 140360 & M137 & 8953 & A & . & 0.00000 & A & & G 0.01451 \\
\hline 147727 & M137 & 16320 & $\mathrm{C}$ & $\mathrm{T}$ & 0.26120 & $\mathrm{C}$ & & $\mathrm{T} 0.05245$ \\
\hline 342450 & M190 & 215 & A & G & 0.08234 & A & & G 0.00097 \\
\hline 597746 & M200 & 8584 & G & A & 0.00541 & G & & A 0.01516 \\
\hline 602733 & M200 & 13571 & $\mathrm{C}$ & $\mathrm{T}$ & 0.01600 & $\mathrm{C}$ & & $\mathrm{T} 0.01060$ \\
\hline 666887 & M203 & 12689 & $\mathrm{~T}$ & $\mathrm{C}$ & 0.00024 & $\mathrm{~T}$ & & C 0.01907 \\
\hline 788952 & M207 & 2746 & $\mathrm{~T}$ & $\mathrm{C}$ & 0.20339 & $\mathrm{~T}$ & & C 0.23444 \\
\hline 1193442 & M236 & 14573 & A & G & 0.30872 & A & & G 0.30129 \\
\hline 1244595 & M240 & 926 & A & G & 0.04277 & A & & G 0.05453 \\
\hline 1302356 & M240 & 11668 & $\mathrm{C}$ & $\mathrm{T}$ & 0.00028 & $\mathrm{C}$ & & Т 0.00041 \\
\hline 1718621 & M480 & 1211 & G & A & 0.02484 & G & & A 0.01028 \\
\hline 1833848 & M490 & 1407 & $\mathrm{~T}$ & $\mathrm{C}$ & 0.00705 & $\mathrm{~T}$ & & C 0.01344 \\
\hline 2046767 & M500 & 204 & $\mathrm{~T}$ & $\mathrm{C}$ & 0.01199 & $\mathrm{~T}$ & & C 0.00118 \\
\hline 2179847 & M512 & 2581 & A & G & 0.01234 & A & & G 0.00043 \\
\hline 2298590 & M520 & 7221 & $\mathrm{~T}$ & $\mathrm{C}$ & 0.00309 & $\mathrm{~T}$ & & C 0.01158 \\
\hline 2382193 & SC8 & 9116 & $\mathrm{~T}$ & $\mathrm{C}$ & 0.01087 & $\mathrm{~T}$ & & C 0.00843 \\
\hline 2497760 & $\mathrm{SC} 16$ & 11149 & G & A & 0.02945 & G & & A 0.02249 \\
\hline 2502781 & $\mathrm{SC} 16$ & 16170 & A & G & 0.06300 & A & & G 0.04775 \\
\hline & ccMinor & CCMAF & cbMajor & cbMinor & cbMAF & ptchange & class & ntchange \\
\hline 140360 & $\mathrm{~T}$ & 0.00037 & A & . & 0.00000 & nonsyn & CDS & ts \\
\hline 147727 & . & 0.00000 & C & $\mathrm{T}$ & 0.00036 & - & Dloop & ts \\
\hline 342450 & G & 0.00122 & A & . & 0.00000 & - & Dloop & ts \\
\hline 597746 & A & 0.00064 & G & . & 0.00000 & nonsyn & CDS & ts \\
\hline 602733 & . & 0.00000 & $\mathrm{C}$ & $\mathrm{T}$ & 0.00049 & nonsyn & CDS & ts \\
\hline 666887 & . & 0.00000 & $\mathrm{~T}$ & $\mathrm{C}$ & 0.00043 & nonsyn & CDS & ts \\
\hline 788952 & A & 0.00031 & $\mathrm{~T}$ & $\mathrm{C}$ & 0.00012 & - & rRNA & ts \\
\hline 1193442 & . & 0.00000 & A & G & 0.00026 & nonsyn & CDS & ts \\
\hline 1244595 & G & 0.00034 & A & . & 0.00000 & - & rRNA & ts \\
\hline 1302356 & $\mathrm{~T}$ & 0.01888 & $\mathrm{C}$ & . & 0.00000 & syn & CDS & ts \\
\hline 1718621 & A & 0.00007 & G & . & 0.00000 & - & rRNA & ts \\
\hline 1833848 & C & 0.00034 & $\mathrm{~T}$ & . & 0.00000 & - & rRNA & ts \\
\hline 2046767 & . & 0.00000 & $\mathrm{~T}$ & C & 0.00127 & - & Dloop & ts \\
\hline 2179847 & C & 0.00045 & A & G & 0.00040 & - & rRNA & ts \\
\hline 2298590 & . & 0.00000 & $\mathrm{~T}$ & C & 0.00126 & nonsyn & CDS & ts \\
\hline 2382193 & C & 0.00030 & $\mathrm{~T}$ & A & 0.00017 & nonsyn & CDS & ts \\
\hline 2497760 & $\mathrm{~T}$ & 0.00015 & G & A & 0.00025 & syn & CDS & ts \\
\hline 2502781 & G & 0.00031 & A & . & 0.00000 & - & Dloop & ts \\
\hline
\end{tabular}

There are a few cases of reversal of mninor allele frequencies between two tissues of the same individual, or between a mother and her child. Consequently, it is necessary to fix the "ancestral" allele, and we arbitrarily decided to use the maternal blood as the ancestral state.

In $[66]: \% R$

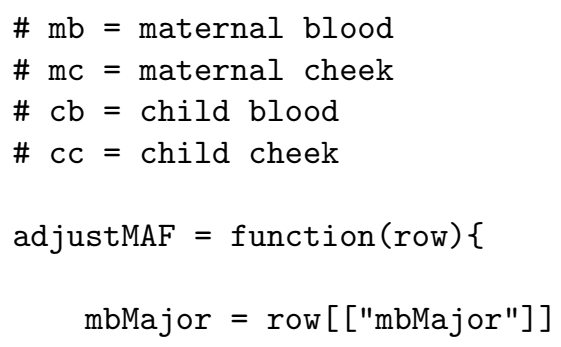




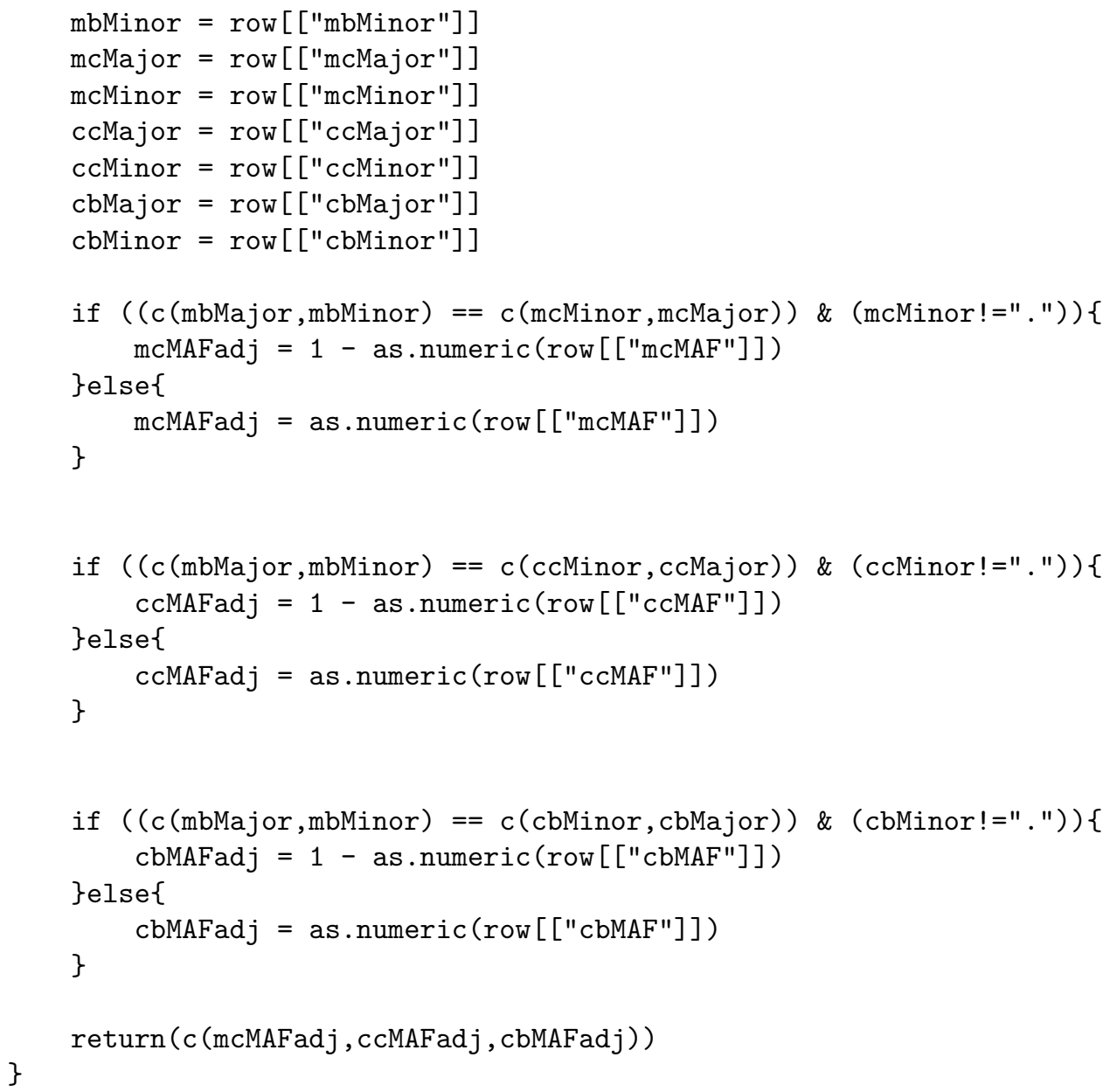

Due to the adjustment of MAF based on the maternal state, comparing the child tissues independently of the mother's, require an additional adjustment.

In $[68]: \% \%$

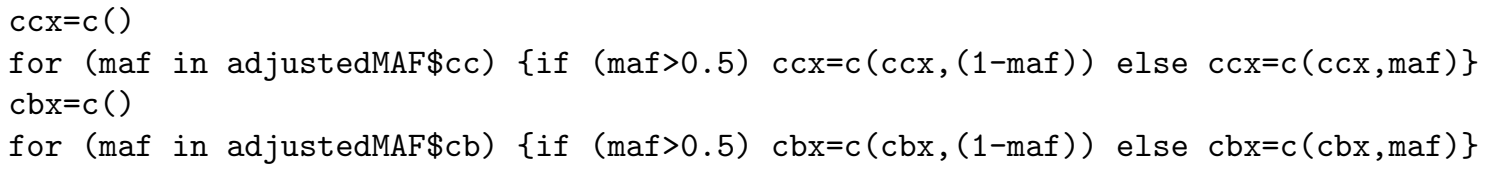



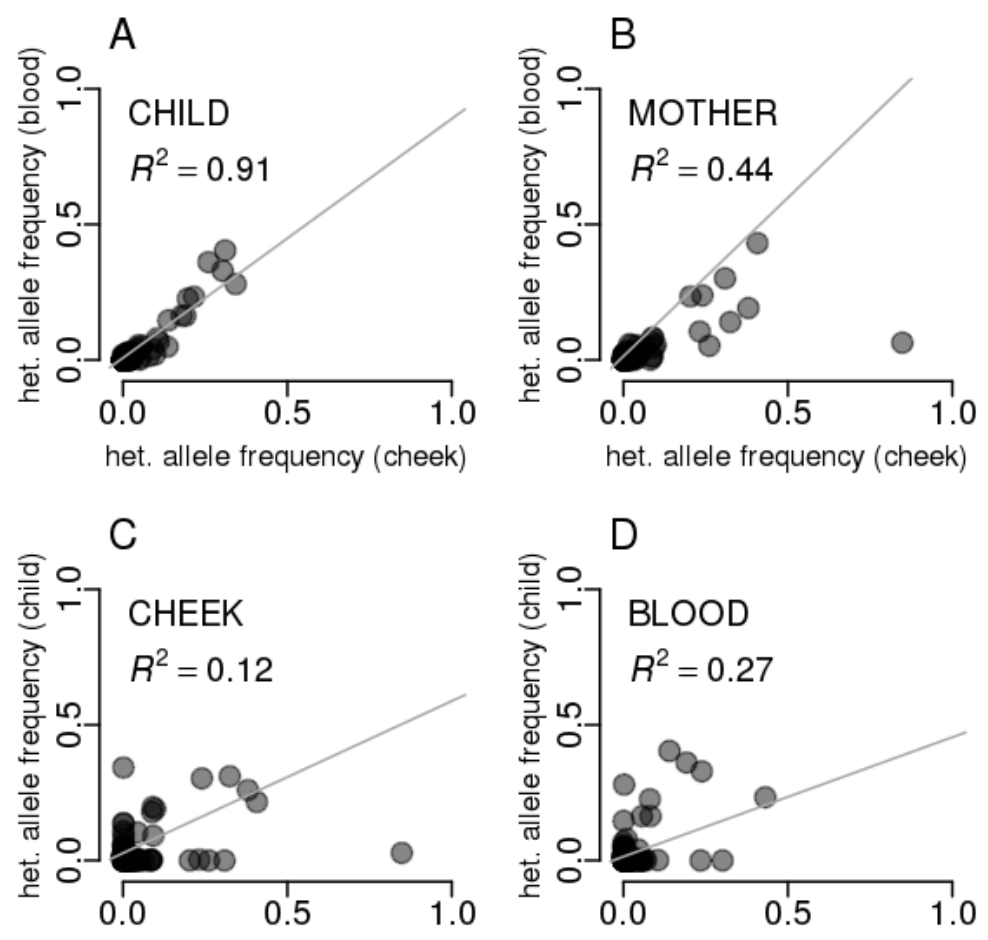

het. allele frequency (mother)

het. allele frequency (mother)

\subsection{Plot the bottleneck size (Fig. S15)}

In $[71]: \% \%$

\# We calculated the bottleneck size by comparing

\# the allele frequency of the minor allele in the mother and her child

bottleneckData = data.frame (

$\mathrm{mc}=$ adjustedMAF $\$ \mathrm{mc}$,

$\mathrm{mb}=$ quartets $[[$ "mbMAF"] $]$,

$c c=a d j u s t e d M A F \$ c c$,

$\mathrm{cb}=$ adjustedMAF $\$ \mathrm{cb}$ )

\# We used the average of the two tissues in an individual

bottleneckData[["meanM"] $]=\operatorname{apply}($ bottleneckData $[, 1: 2], 1$, mean)

bottleneckData $[$ [meanC"] $]=\operatorname{apply}($ bottleneckData $[, 3: 4], 1$, mean)

\# And modeled the bottleneck as in Millar et al, 2008

bottleneckData\$bn1 = (bottleneckData\$meanM*(1-bottleneckData\$meanM)) / (bottleneckData $\$$ meanC-bot bottleneckData $\$$ bn 1. cheek = (bottleneckData $\$$ mc* $(1-$ bottleneckData $\$ \mathrm{mc})) /($ bottleneckData $\$ \mathrm{mc}-\mathrm{bottlen}$ bottleneckData $\$$ bn 1 .blood = (bottleneckData\$mb*(1-bottleneckData $\$ \mathrm{mb})) /($ bottleneckData $\$ \mathrm{mb}-\mathrm{bottlen}$ 
\# Select cases where there is evidence of the minor allele in the maternal linage (i.e. the mi

\# both tissues of the mother, at least $1 \%$ in one of the tissues, and $0.2 \%$ in the other tissue)

bn1.m = bottleneckData $[$ (bottleneckData $\$ \mathrm{mc}>=0.01 \mid$ bottleneckData $\$ \mathrm{mb}>=0.01) \quad \&$ (bottleneckData $\$ \mathrm{mc}>=0.002$ \& bottleneckData $\$ \mathrm{mb}>=0.002)],[[" \mathrm{bn} 1 "]]$

bn1. cheek = bottleneckData[bottleneckData\$mc $>=0.01],[[$ "bn1. cheek"] $]$

bn1.blood = bottleneckData[bottleneckData $\$ \mathrm{mb}>=0.01],[[" \mathrm{bn} 1 . \mathrm{blood} "]]$

In $[72]: \% \mathrm{R}$

length (bn1.m)

[1] 50

In $[73]: \% \%$

\# Accounting for mitotic segregation

mitotic $=$ function $($ row $)\{$

$\mathrm{mc}=\operatorname{row}[1]$

$\mathrm{mb}=\operatorname{row}[2]$

$\mathrm{cc}=\operatorname{row}[3]$

$\mathrm{cb}=\operatorname{row}[4]$

variance $=\left((m c-c c)^{\wedge} 2+(m c-c b)^{\wedge} 2+(m b-c c) \wedge 2+(m b-c c)^{\wedge} 2-2 *(m c-m b)^{\wedge} 2-2 *(c c-c b) \wedge 2\right) / 4$

return (variance)

\}

bottleneckData[["mitotvar"]] = apply(bottleneckData,1,mitotic)

bottleneckData\$bn2 = (bottleneckData\$meanM* (1-bottleneckData\$meanM))/(bottleneckData\$mitotvar)

bn2.m = bottleneckData $[$ (bottleneckData $\$ m c>=0.01 \mid$ bottleneckData $\$ m b>=0.01) \&$

(bottleneckData\$mc > =0.002 \& bottleneckData\$mb>=0.002), [ ["bn2"]]

In $[74]: \% R$

\# We removed negative or indetermined estimates of the bottleneck

$\mathrm{bn} 2 \cdot \mathrm{m}=\mathrm{bn} 2 \cdot \mathrm{m}[\mathrm{bn} 2 \cdot \mathrm{m}>0]$

In $[75]: \% \mathrm{R}$

length (bn2.m)

[1] 45

In $[76]: \% \mathrm{R}$

bn1.m

[1] $\quad 23.816855 \quad 359.631901$

[6] $\quad 26.075053 \quad 107.006629$

5.388898

66.758429

23.645718

[11] $\quad 103.069255$

23.161960

5.081444

102.508491

76.998633

[16] 4576.759055

87.090388

66.648376

3.575002

5.999405

$\begin{array}{lll}7.145162 & 543.823984 & 681.303195\end{array}$ 


$\begin{array}{lrrrrr}{[21]} & 2.280578 & 30.658853 & 19.692368 & 286.394261 & 12.206464 \\ {[26]} & 20.436016 & 10.789702 & 56.171303 & 99.896336 & 68.929415 \\ {[31]} & 10199.573616 & 81.633597 & 162.572697 & 20.286884 & 31.950263 \\ {[36]} & 4.906696 & 1.059908 & 1.253351 & 32.734057 & 564.244306 \\ {[41]} & 63.027094 & 48.650744 & 161.959066 & 1.290042 & 107.814053 \\ {[46]} & 6.428074 & 38.090392 & 17.154591 & 103.400598 & 88.887083\end{array}$

In $[77]: \% \%$

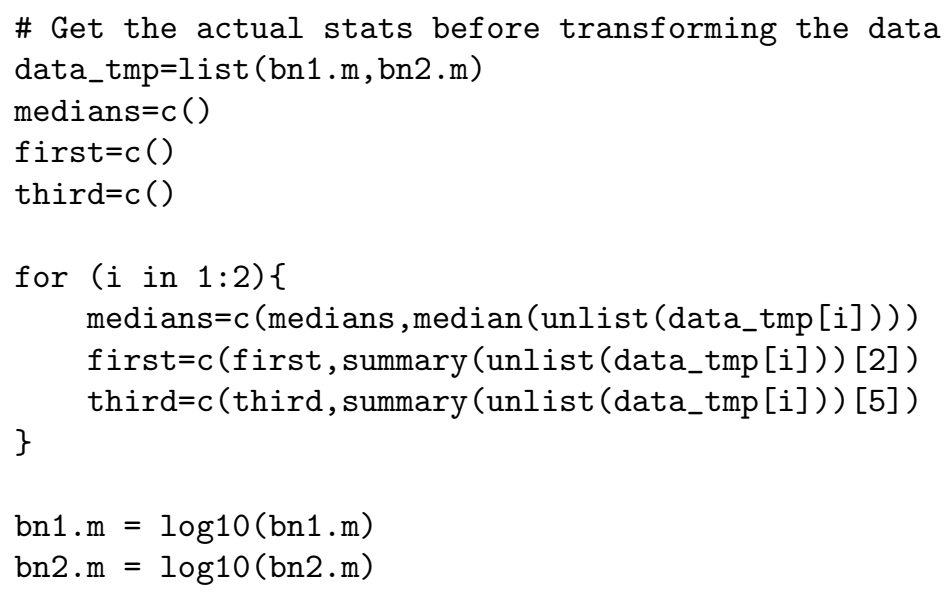



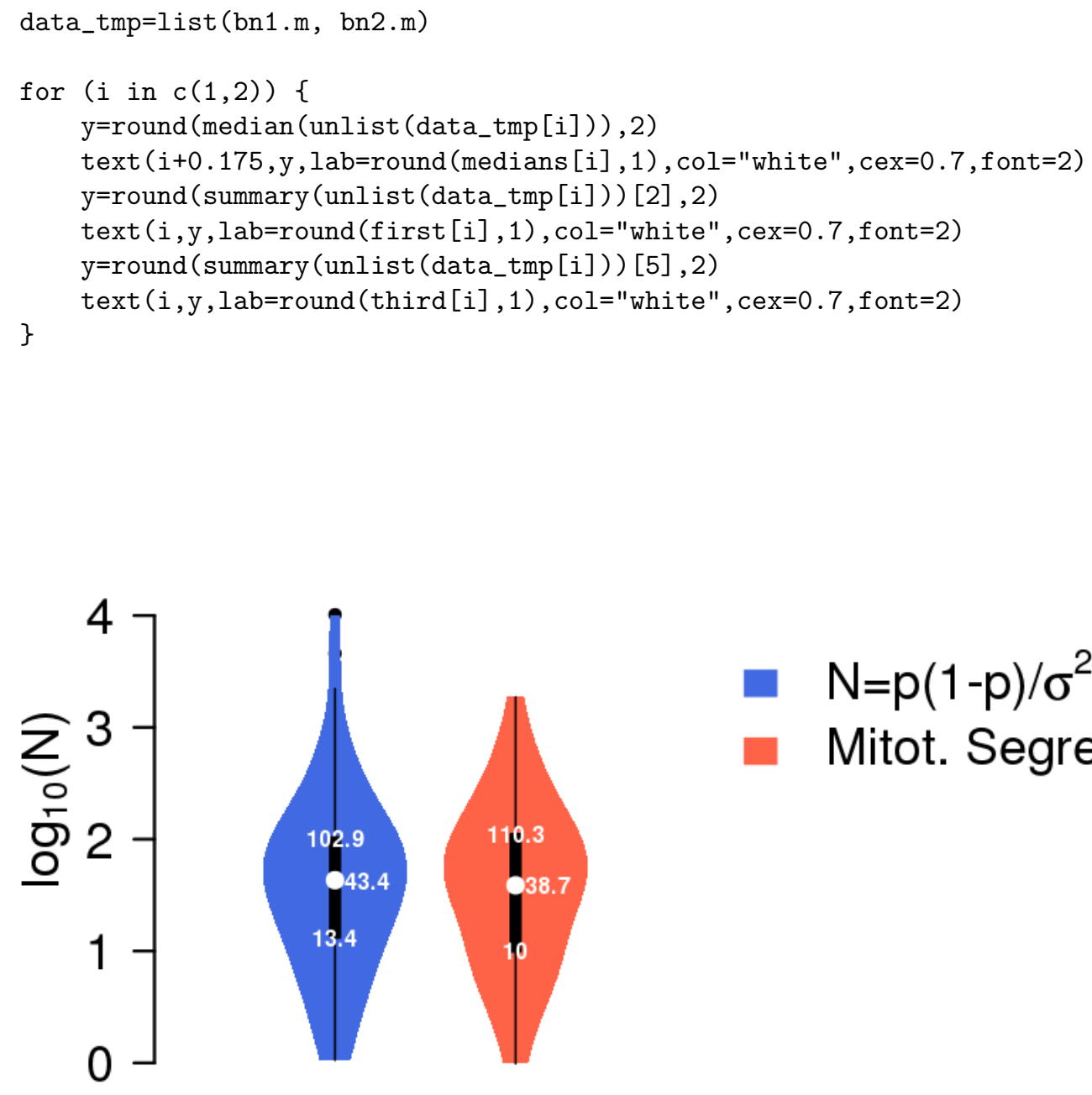

$N=p(1-p) / \sigma^{2}$ gen
Mitot. Segreg.

\subsection{Plot age correlations (Fig. 2)}

In $[79]: \% \%$

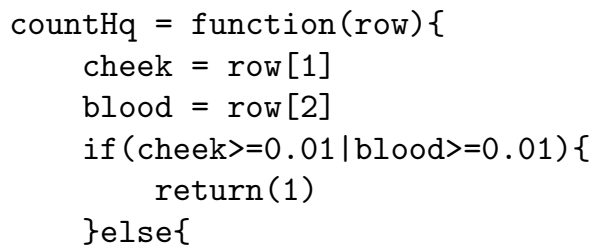




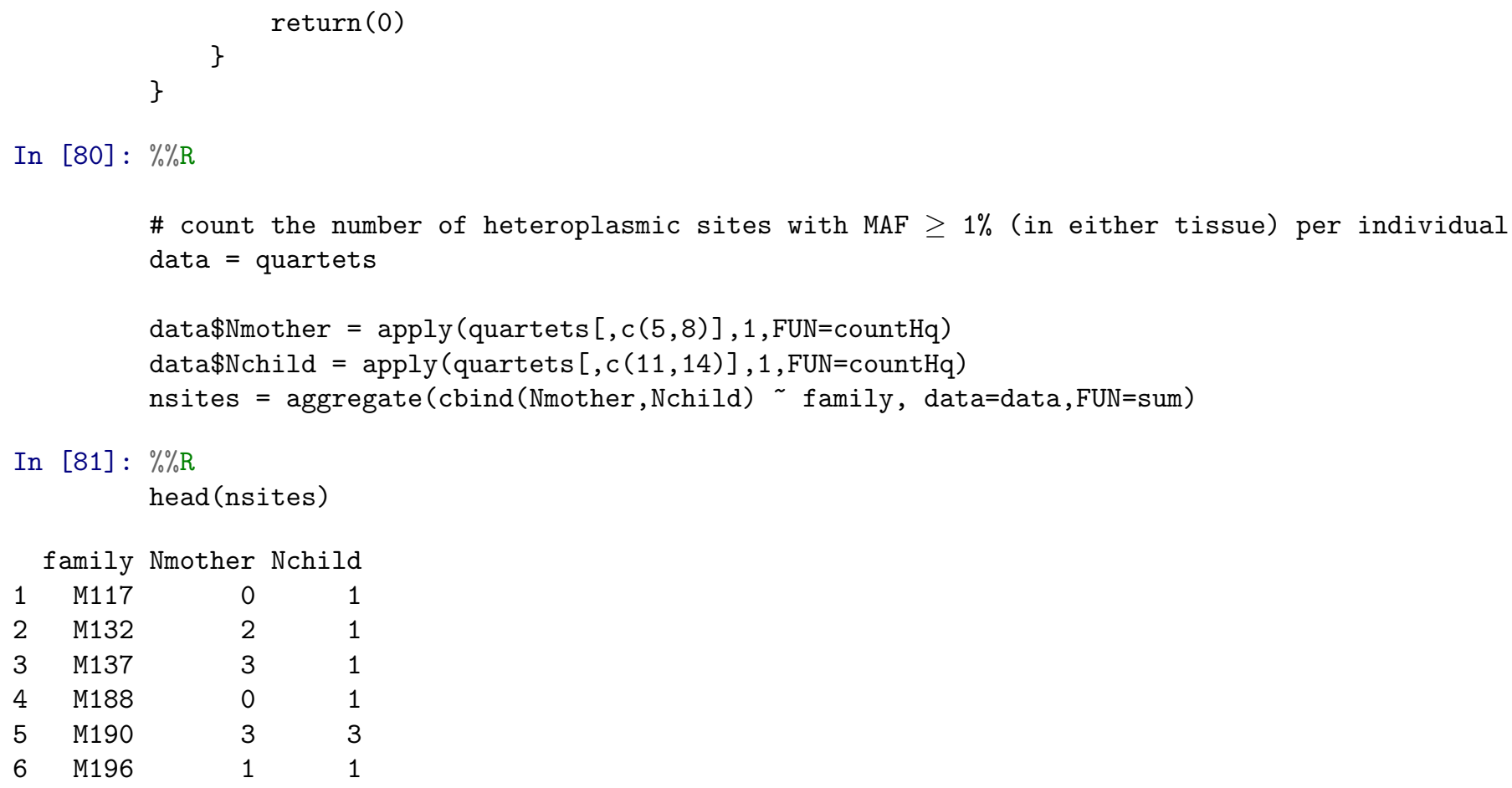

\begin{tabular}{rrrrr}
\multicolumn{5}{c}{ \# age in days } \\
head(sampAges $)$ \\
mother motherAgeCollection & child & childAgeCollection \\
0 & M132 & 16658 & M132C1 & 7460 \\
1 & M137 & 14294 & M137C2 & 6202 \\
2 & M186 & 15938 & M186C2 & 3504 \\
3 & M188 & 17714 & M188C2 & 3451 \\
4 & M190 & 18761 & M190C2 & 5866 \\
5 & M195 & 11826 & M195C1 & 2752
\end{tabular}

In $[84]: \% \%$

ageEffect $=$ merge (nsites, sampAges, by $\cdot \mathrm{x}=$ "family", by $\cdot \mathrm{y}=$ "mother", all $\cdot \mathrm{y}=\mathrm{TRUE})$

\# for samples without heteroplasmic sites, merging produces NAs, so we transformed them to zer ageEffect [is.na(ageEffect)] = 0

In $[85]: \% \%$

\# the age of the mother at the time of conception of the child is assumed to be \# the current age of the mother, less the current age of the child, less nine months (in days ageEffect $[[$ "motherAgeFertilization"] $]=\operatorname{ageEffect}[[$ "motherAgeCollection"] $]$ - (ageEffect [["chil 
In [86]: $\% \%$ R $-\mathrm{w} \quad 4-\mathrm{h} \quad 4-\mathrm{u}$ in $-\mathrm{r} 144$

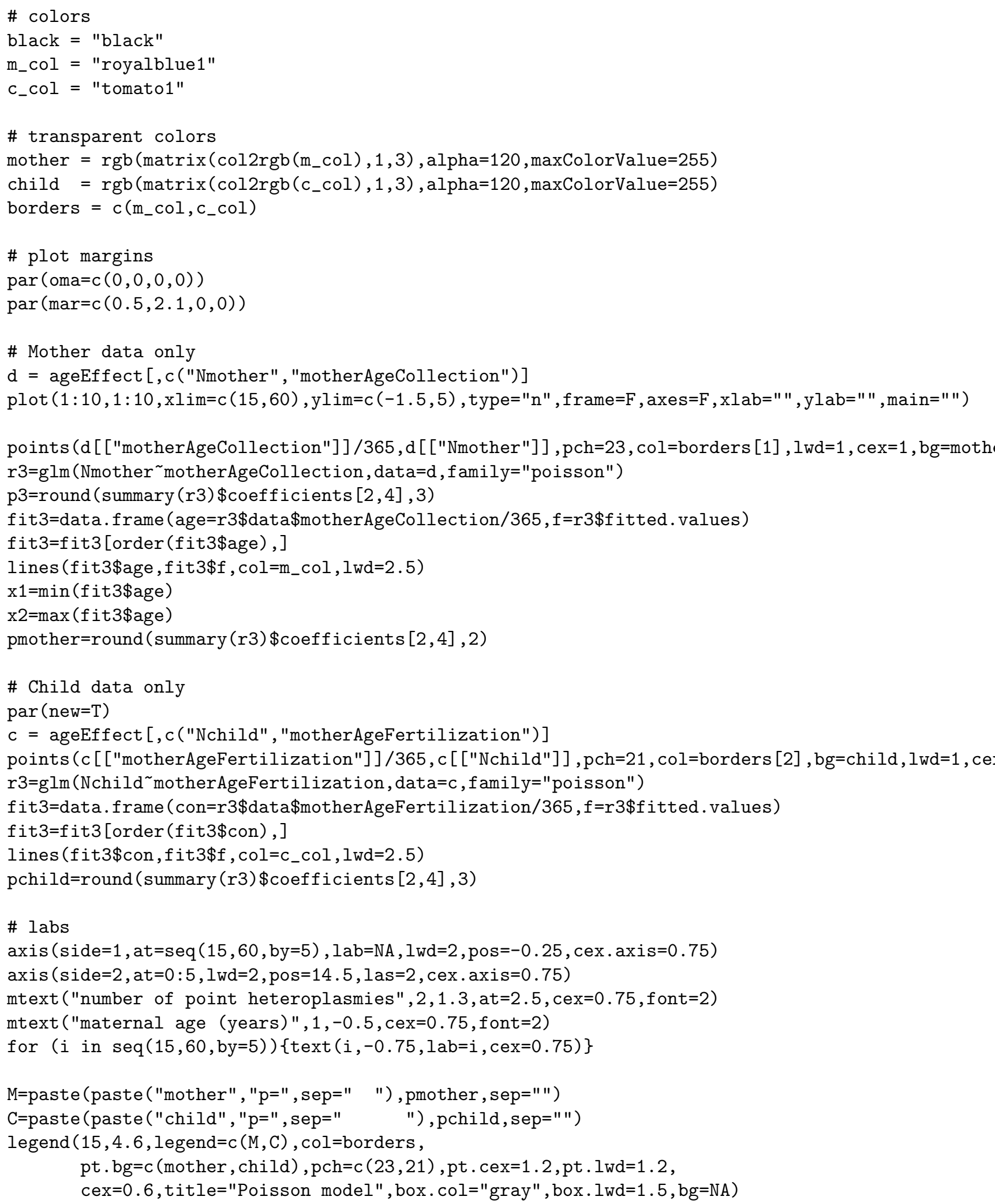




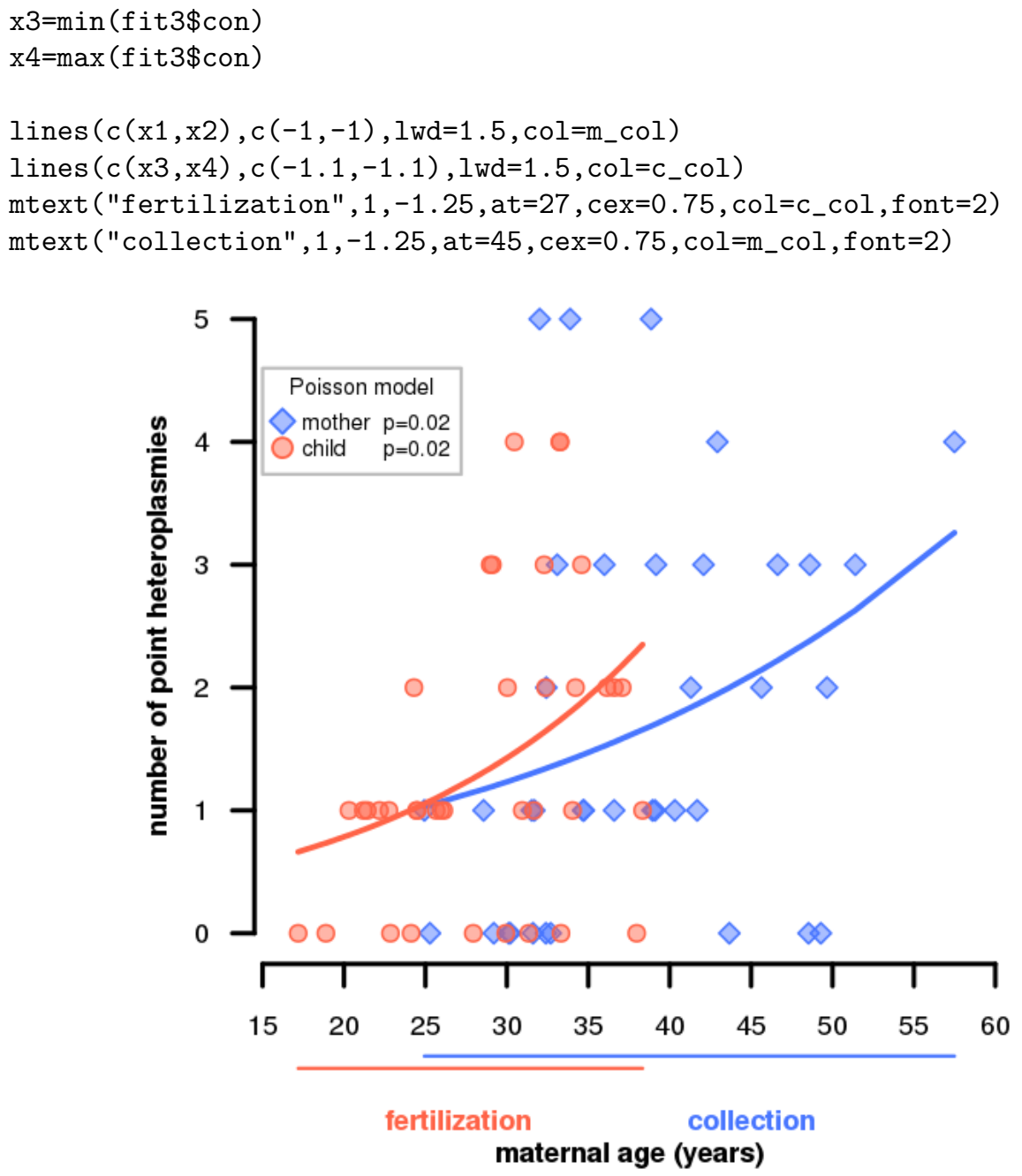

In [ ]: 